\title{
Measuring and Visualizing Chlamydia and Gonorrhea Inequality: An Informatics Approach Using Geographical Information Systems
}

Patrick T.S. Lai*, M.P.H. ${ }^{1}$, Jeffrey Wilson, Ph.D ${ }^{2}$, Huanmei $\mathbf{W u}^{1,3}$, Ph.D, Josette Jones ${ }^{1}$, Ph.D, Brian E. Dixon, Ph.D., MPA 4,5

1. School of Informatics and Computing, Indiana University, Indianapolis, IN

2. School of Liberal Arts, Department of Geography, Indiana University, Indianapolis, IN

3. Purdue School of Engineering and Technology, Purdue University, Indianapolis, IN

4. Richard M. Fairbanks School of Public Health, Indiana University, Indianapolis, IN

5. Regenstrief Institute, Inc., Center for Biomedical Informatics, Indianapolis, IN, USA

\begin{abstract}
Background: Health inequality measurements are vital in understanding disease patterns in identifying high-risk patients and implementing effective intervention programs to treat and manage sexually transmitted diseases.

Objectives: To measure and identify inequalities among chlamydia and gonorrhea rates using Gini coefficient measurements and spatial visualization mapping from geographical information systems. Additionally, we seek to examine trends of disease rate distribution longitudinally over a ten-year period for an urbanized county.

Methods: Chlamydia and gonorrhea data from January 2005 to December 2014 were collected from the Indiana Network for Patient Care, a health information exchange system that gathers patient data from electronic health records. The Gini coefficient was used to calculate the magnitude of inequality in disease rates. Spatial visualization mapping and decile categorization of disease rates were conducted to identify locations where high and low rates of disease persisted and to visualize differences in inequality. A multiple comparisons ANOVA test was conducted to determine if Gini coefficient values were statistically different between townships and time periods during the study.

Results: Our analyses show that chlamydia and gonorrhea rates are not evenly distributed. Inequalities in disease rates existed for different areas of the county with higher disease rates occurring near the center of the county. Inequality in gonorrhea rates were higher than chlamydia rates. Disease rates were statistically different when geographical locations or townships were compared to each other ( $p$ $<0.0001)$ but not for different years or time periods $(p=0.5152)$.

Conclusion: The ability to use Gini coefficients combined with spatial visualization techniques presented a valuable opportunity to analyze information from health information systems in
\end{abstract}


investigating health inequalities. Knowledge from this study can benefit and improve health quality, delivery of services, and intervention programs while managing healthcare costs.

Keywords: Sexually transmitted diseases, Data analysis, Data visualization, Health inequalities, Gini coefficient

Abbreviations: Geographical information systems (GIS), Sexually transmitted diseases (STDs), American Community Survey (ACS), Indiana Network for Patient Care (INPC), Local indicators for spatial autocorrelation (LISA)

*Correspondence: Patrick T.S. Lai ptlai@iupui.edu

DOI: 10.5210/ojphi.v11i2.10155

Copyright @2019 the author(s)

This is an Open Access article. Authors own copyright of their articles appearing in the Online Journal of Public Health Informatics. Readers may copy articles without permission of the copyright owner(s), as long as the author and OJPHI are acknowledged in the copy and the copy is used for educational, not-for-profit purposes.

\section{INTRODUCTION}

Health inequality, a term that describes an adverse difference in health among advantaged and disadvantaged groups in a population, is a serious, ongoing, and persistent problem that negatively affects the health of populations in the United States [1,2]. The consequences of health inequalities are detrimental as it leads to increased mortality and sickness, increased health care costs, decreased productivity, and lower control over personal health and development [3,4]. With the presence of health inequalities, not everyone is benefiting from the advances in healthcare and medical treatment that is essential in maintaining well-being and optimal health. Different subsets of the population remain highly vulnerable to disease risk and infection, and various geographical areas have extreme inequalities in disease rates that require attention and focus. The need to identify high risk areas remains paramount for clinicians to effectively locate vulnerable populations in the effective management and treatment of disease. More importantly, there is a necessity to establish accurate methods to measure health disparity and inequality for identifying and creating policy interventions, prioritizing essential health resources, and understanding how communicable diseases are transmitted [5]. Because of this, analysis of health inequalities remains a critical focal point for clinical health research especially when monitoring trends in disease risk, establishing novel research methodologies, and creating interventions that improve the current health status of the population.

One method to measure inequality is the Gini coefficient or Gini index. In 1912, an Italian economist by the name of Corrado Gini developed the Gini coefficient to measure statistical dispersion in representing the distribution of data [6]. When the Gini coefficient was first established, its initial sole purpose was to measure the magnitude and degree of inequality in income distribution within a population which was represented with a numeric value between 0 and 1 . Values closer to 0 reflect greater equality or uniformity in income distribution while values closer to 1 reflect greater magnitudes of inequality [7]. This coefficient can also be visualized graphically on an $x-y$ graphical plot using the Lorenz curve which is a line that represents the 
cumulative proportion of income ( $\mathrm{y}$-axis) versus the cumulative proportion of the population ( $\mathrm{x}$ axis) as shown in Figure 1 [8]. On the same plot, a hypothetical diagonal line of equality is drawn to represent perfect equality of income. The Gini coefficient represents twice the area between the line of equality and the Lorenz curve. Higher Gini coefficients representing greater inequality present Lorenz curves that deviate the furthest away from the line of equality while the smallest Gini coefficients present Lorenz curves that are closest to the line of equality [9]. Thus, the Gini coefficient provides a convenient way to calculate the uniformity or concentration of values in a dataset.

The value and usefulness of the Gini coefficient measurement of inequality can greatly be expanded by identifying and locating where the highest inequalities persist and where they reside. Spatial data visualization techniques and geographic information systems (GIS) can assist in locating inequalities as they can visualize the distribution of the data and detect anomalies contained in the data of interest [10]. Furthermore, using these spatial data visualization tools can also aid in the ability to examine relationships and patterns of different health outcomes and the determinants that influence these outcomes [11]. As a benefit, these tools generate new information about disease risk where data is limited or where there is a lack of disease surveillance [12]. As a result, spatial data visualizations create health information about geographical locations that is easy to analyze, interpret, and organize [13].

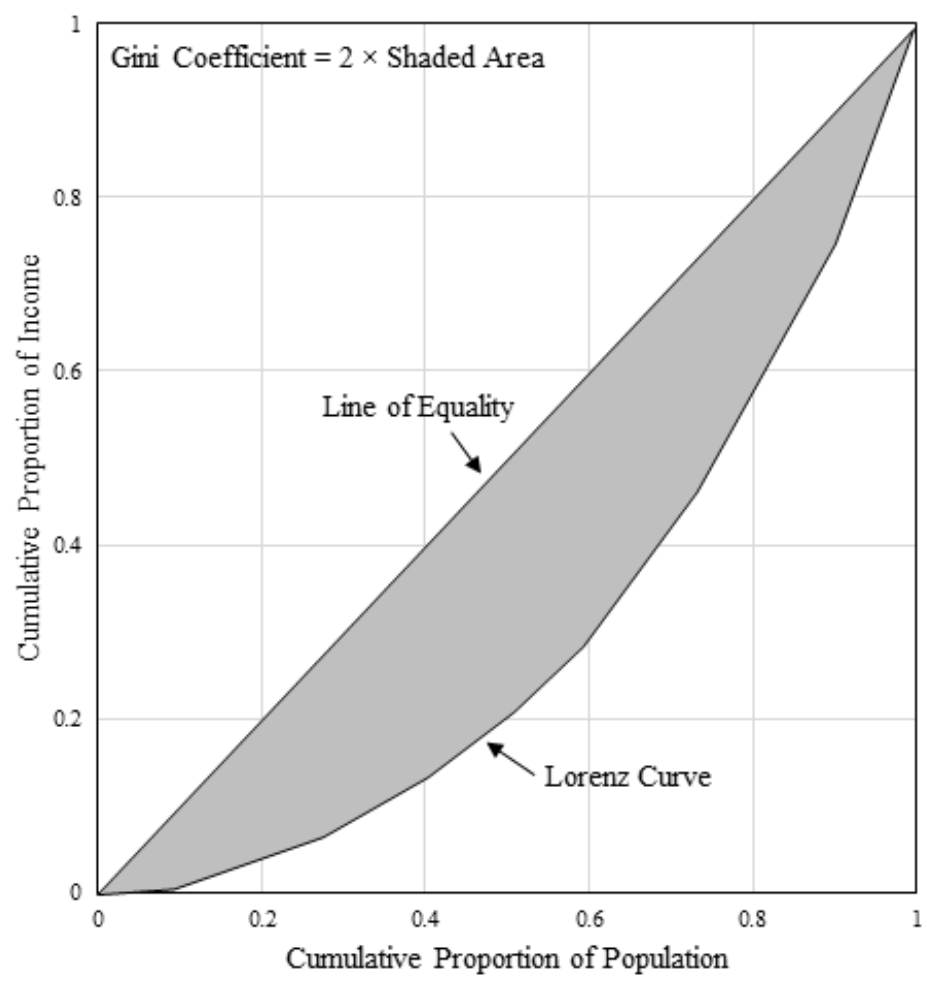

Figure 1 Graphical representation of the Lorenz Curve

The Gini coefficient itself has been widely used initially in the field of economics to measure income distribution, but it has also been applied to other applications outside of economics. In 
geography for example, the Gini coefficient was applied in the measurement of cluster size for spatial clustering and concentration [14]. For healthcare and medicine, the Gini coefficient was utilized to analyze the relationship between health disparities and income inequality, measure fairness in health ethics and equity, calculate the distribution of healthcare personnel, and analyzing trends of geographical disparities in health care provision [9,15-17]. A limited number of studies have incorporated the use of the Gini coefficient in the study of sexually transmitted diseases. In an article from Althaus et al., authors used the Gini coefficient to measure the distribution of chlamydia infections in determining sexual behavior in Great Britain [18]. Another study from Leichliter et al. also incorporated the usage of the Lorenz curve and Gini coefficient to measure the concentration of sexual behaviors and sexual partners by gender and race using a probability sample of the United States population [19]. One such study from Kerani et al. using data from non-electronic disease reporting gave a comprehensive approach of measurement with the Gini coefficient to compare concentration levels and clustering between four different sexually transmitted diseases (syphilis, chlamydia, gonorrhea, and genital herpes) for King County, Washington in 2005 [20]. Their study concluded that among the four diseases, genital herpes had the lowest concentration of cases followed by chlamydia, gonorrhea, and syphilis having the highest concentration. Because of these studies, the Gini coefficient presents many applications and has become quite versatile in analyzing and understanding the distribution of various information.

When conducting health disparity research, the routine investigation of sexually transmitted diseases (STDs) particularly chlamydia and gonorrhea presents an excellent opportunity to analyze and measure health inequalities using the Gini coefficient. Untreated STD infections can lead to serious health conditions and complications such as blindness, infertility, pelvic inflammatory disease, inflammation of reproductive organs, and possible cervical cancer in women [21-25]. Numerous challenges exist to effectively control and reduce the transmission of STDs with the presence of health inequalities being a prime factor. In many instances, chlamydia and gonorrhea rates are unevenly distributed geographically leading to areas where disease rates occur disproportionally resulting in segments of the population to be much more susceptible to infection. This creates a challenge on where and to what degree interventions should be placed for these susceptible or high-risk groups. Besides the research by Kerani et al., no other studies have examined or measured the distribution and inequality of both chlamydia and gonorrhea disease rates over time using the Gini coefficient at the county level [20]. Additionally, there are very few studies that incorporated the ability to visualize these inequalities in accordance with such inequality measures. Our study on chlamydia and gonorrhea inequality provides a novel approach to present how the combination of inequality measures and spatial data visualizations can be used to locate and quantify the degree of disease inequality from clinical data sources.

In our study, we seek to accomplish three goals in understanding inequality among chlamydia and gonorrhea disease rates in an urbanized county in central Indiana using longitudinal data. First, we seek to quantify the magnitude of inequality among different areas of the county using the Gini coefficient. Secondly, we seek to identify and locate areas across the county with the greatest inequality of disease rates using spatial data visualization techniques. Finally, we aim to determine if there are differences in chlamydia and gonorrhea disease rates over a ten-year period and by geographical locations within the county. The main outcome of this paper will assist healthcare 
professionals to identify disease patterns and understand distributions of health inequality in sexually transmitted diseases.

\section{METHODS}

\section{Setting}

The geographical area of interest in this study is Marion County, Indiana, which is the location of the major capital city of Indianapolis along with several smaller incorporated cities. The county has a total area of 403.01 square miles and a total population of 919,336 as of 2014 according to the American Community Survey (ACS) 5-year estimates [26]. The county is comprised of a total of 9 townships as depicted in Figure 2 with a total of 224 separate census tracts. The townships starting from northwest to southeast with the number of census tracts are Pike $(\mathrm{n}=17)$, Washington $(n=37)$, Lawrence $(n=23)$, Wayne $(n=35)$, Central $(n=55)$, Warren $(n=23)$, Decatur $(n=5)$, Perry $(n=21)$, and Franklin $(n=8)$. The downtown core area of the city of Indianapolis is located in Central Township. According to the United States Census Bureau, a census tract is defined as a geographical feature that is comprised of about 1,200 to 8,000 people, a characteristic roughly similar to the size of a neighborhood [27]. The boundaries of census tracts are considered relatively permanent subdivisions of geographical units for statistics and population enumeration. Multiple census tracts can be grouped together to form a township which represent subdivisions of a county.

Marion County and the Indianapolis metropolitan area had one of the highest disease burdens of chlamydia and gonorrhea infection according to the Centers for Disease Control and Prevention. The reported rate of chlamydia increased from 605.7 cases to 683.6 cases per 100,000 population while the reported rate of gonorrhea also increased from 185.1 cases to 270.9 cases per 100,000 population during the time period between 2013 to 2017 [28,29]. These rates are much higher than the average rates for all major metropolitan areas studied. As a result, this geographical region has consistently been ranked in the top six in terms of highest chlamydia and gonorrhea disease rates among all major metropolitan areas.

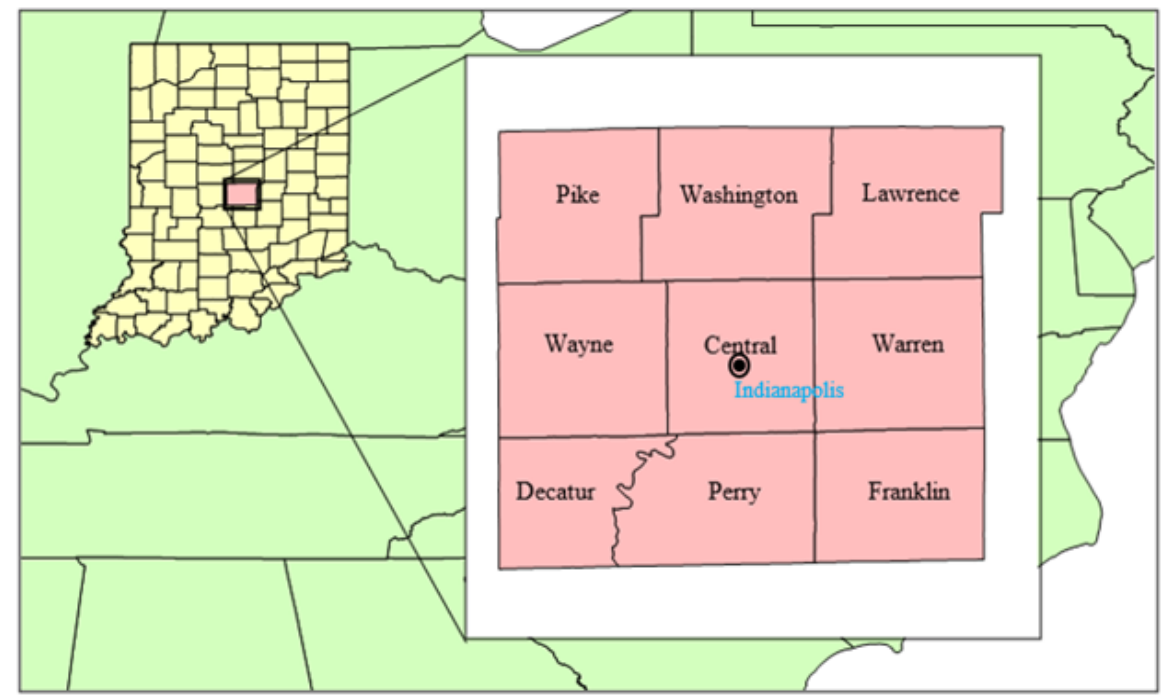

Figure 2 Geographical representation of Marion County, Indiana with the nine townships 


\section{Data Source and Computation of Disease Rates}

Clinical data on chlamydia and gonorrhea cases were extracted from the Indiana Network for Patient Care (INPC), a large health information exchange system network that collects and manages electronic health record data from over 100 participating hospitals and 38 health systems within Indiana [30,31]. Healthcare entities, hospitals, and providers who participate in this system submit their reports and patient information to the INPC. A case of chlamydia and gonorrhea is defined as an individual with a positive laboratory test for the disease [32]. If an individual has two positive confirmed tests that are more than thirty days apart (> 30 days), these tests were counted as two separate cases [33]. As both chlamydia and gonorrhea are considered notifiable diseases, hospitals and providers are required to report these diseases to public health within seventy-two hours as mandated by Indiana law [34]. A period of ten years of chlamydia and gonorrhea positive cases were retrospectively collected from January 1, 2005 to December 31, 2014 from the INPC. Each positive case was linked to the census tract of the corresponding patient's residential address to ensure patient confidentiality. Positive cases that were not able to be geocoded or had an undetermined address were excluded in this study. The number of positive cases in the census tracts were aggregated together to create a total sum of cases for each of the nine townships. Similarly, the number of unique individuals with a record in the INPC system was tabulated for each census tract and are grouped together for each township. This is referred as the INPC population. If a patient had multiple visits with a provider who participated in the INPC in the same year, they will still be counted as one unique record in the INPC. The total numbers of chlamydia and gonorrhea cases that were geocoded and used in this study are tabulated in a frequency table including the number of individuals with a unique INPC record for each year.

To compute chlamydia and gonorrhea incidence rates, we use the number of positive cases as the numerator divided by the number of unique individuals with a record in the INPC system as the denominator for that given year. This computation of rates was applied for both census tract and township levels. For each township, the lowest and highest rates of chlamydia or gonorrhea were recorded with the descriptive statistics for chlamydia and gonorrhea rates.

\section{Visualization of Disease Rates}

Chlamydia and gonorrhea disease rates for each census tract were represented using a choropleth map for chlamydia and gonorrhea. Darker colors represented higher rates of disease while lighter colors represented lower rates of disease. To depict the visualization of disease rate disparity across the county, individual census tracts were grouped into ten deciles in order from lowest rates to highest rates separately for each disease. The first decile group represented census tracts with the lowest $10 \%$ of disease rates (dark blue) while the tenth decile group represented census tracts with the highest $10 \%$ of disease rates (dark red). Census tracts with moderate disease rates or middle decile groups are represented with lighter colors. Mapping of disease inequality was conducted for all ten years of study from 2005 to 2014. All spatial data visualizations were created using ArcGIS Desktop 10.6. 


\section{The Gini Coefficient to Analyze and Measure Inequality}

We apply the methodology of the Lorenz curve from an income inequality perspective to the inequality of disease rates in a geographical area. In our study, the Lorenz curve is the line that represents the cumulative proportion of either chlamydia or gonorrhea disease cases (y-axis) versus the cumulative proportion of the population (x-axis). A diagonal line of equality represents a complete uniform distribution of disease rates. A Lorenz curve that is flatter or closer to the line of equality would result in more uniformity of disease rates in the context of disease. A Lorenz curve that is furthest away from the diagonal line of equality signifies increasing inequality or disparity in disease rates over a geographical area. This could represent that the rate of disease is highly variable in the population and is highly concentrated in a specific area of a region resulting in higher inequality [35].

The Gini coefficient examines the degree of departure from a uniform equal distribution of values and to determine inequality among the values. To calculate the Gini coefficient for each township, census tracts were ordered from the lowest to highest rates within each township for each disease. From the ordered census tracts, the number of cases were added together to give the cumulative proportion of cases starting with the census tracts with zero cases followed by census tracts with one case, and so forth. The INPC population from these ordered census tracts were also aggregated together to determine the cumulative proportion of population. Thus, the Gini coefficient was calculated using the formula:

$$
G=\frac{1}{2} \sum_{i=1}^{k}\left|X_{i}-Y_{i}\right|
$$

where $X_{i}$ is the proportion of positive disease cases, $Y_{i}$ is the proportion of the INPC population, $k$ is the number of census tracts in a township, and $G$ is the Gini coefficient [15]. The Gini coefficient was calculated as twice the area between the diagonal line of equality and the Lorenz curve as depicted in Figure 1 [36]. This approach to measure inequality of sexually transmitted disease cases has been adopted using a similar methodology from a previous study by Kerani et al. [20] Gini coefficients closer to 0 represent greater equality and uniformity among disease rates while Gini coefficients closer to 1 represent significant inequality where only a small specific group in a population receive a very large amount of disease cases in a geographical area. Mathematical calculations of all Gini coefficients were performed using a programmable statistical software called RStudio version 1.1.442 using the DescTools package.

\section{Statistical Analyses}

To determine if there is a difference in the inequality of disease rates by year and by township for both diseases, we administered a one-way ANOVA analysis along with a post-hoc multiple comparisons test to determine which years and townships are statistically different from each other. In performing this analysis, we used a longitudinal collection of data spanning ten years to determine the trends of chlamydia and gonorrhea disease rates from 2005 to 2014. All $p$-values were adjusted using the Tukey correction to limit the Type I error involved in conducting multiple 
t-tests with the alpha level starting at $\alpha=0.05$. Statistical analyses for the one-way ANOVA and multiple comparisons test were performed using SAS 9.4 software.

\section{RESULTS}

Table 1 enumerates the frequency counts of chlamydia and gonorrhea cases along with the total number of individuals who were in the Indiana Network for Patient Care (INPC) health information exchange system between 2005 to 2014 for each township in Marion County. Most of the cases for chlamydia and gonorrhea were found in Central Township over the ten-year period while Decatur and Franklin Townships on the southernmost portion of the county had the least number of cases. There is also a slight upward trend in the number of cases recorded for chlamydia from 2005 to 2012 and gonorrhea from 2005 to 2011 followed by a downward trend for both diseases. The descriptive statistics and characteristics of chlamydia and gonorrhea rates for each township between 2005 to 2014 are presented in Table 2. In general, the highest rates for both diseases were found in Central Township while the lowest rates of disease were observed in the southernmost townships of Decatur, Perry, and Franklin. For the county, the disease rates showed a slightly downward trend for chlamydia from 2005 to 2010 followed by an increasing upward trend starting in 2011. For gonorrhea, there was no distinctive trend occurring as the rates were observed to fluctuate over the ten-year period. The overall gonorrhea rates, however, are indeed lower compared to chlamydia rates.

Spatial data visualizations of chlamydia and gonorrhea incidence rates are represented in Figure 3 and Figure 4, respectively for the ten-year period. Furthermore, a different approach to visualization was applied to identify and examine disease rate inequality by using decile grouping of disease rates as shown in Figure 5 and Figure 6. From these visualizations, many of the highest disease rates were situated in the central part of the county, particularly along the border between south Lawrence-north Warren, south Washington-north Central, and southeast Pike-northeast Wayne Townships for both diseases. These areas contained census tracts that were ranked in the highest decile groups for disease rates (dark red). Conversely, the lowest disease rates were predominantly found on the outer edges of the county including southeast Franklin and northeast Lawrence Townships. These areas were characterized as having census tracts that were ranked in the lowest decile groups for disease rates (dark blue). Middle decile groups representing moderate chlamydia or gonorrhea disease rates (lighter colors) were mostly scattered throughout the county with no distinct pattern or consistency. Central Township had the most census tracts with higher decile groups of disease rates while Decatur, Perry, and Franklin Townships exhibited the least amount. However, a few census tracts exhibiting moderate disease decile groups were observed in the three southernmost townships for gonorrhea. Some townships particularly Washington and Lawrence had a distinctive gradient in having census tracts with the highest decile groups in the southernmost portion of the townships and the lowest decile groups in the northernmost portion of the townships. This finding was evident for both diseases which may signify high inequality in disease rates within those townships.

Each of the ten decile groups from 2005 to 2014 was quantified for the percentage of population and the percentage of cases within each decile group. The results are found in Table 3 for chlamydia and gonorrhea. Table 3 also depicts the Gini coefficient inequality of disease rates for the entire county. For most of the years, the highest decile group (Highest 10\%) had roughly 19.1\% 
to $23.7 \%$ of all chlamydia cases within the county while the lowest decile group (Lowest $10 \%$ ) contained between $1 \%$ to $1.6 \%$ of all cases. The top half which represents the $6^{\text {th }}$ decile to the highest $10 \%$ decile groups had about half of the population in the county but contained roughly $73.6 \%$ to $77.8 \%$ of all chlamydia cases. The disparity between the highest decile and lowest decile group for gonorrhea was even greater where the highest decile group had roughly $23.1 \%$ to $26.6 \%$ of all cases while the lowest decile group contained $0.3 \%$ to $0.8 \%$ of all cases. Furthermore, the Gini coefficients over the ten-year period for gonorrhea were higher than the Gini coefficients for chlamydia. The overall Gini coefficient range of inequality for chlamydia was from 0.344 to 0.392 while the range of inequality for gonorrhea was from 0.417 to 0.454 . For both diseases, there were no large fluctuations or variability in the Gini coefficients from year to year. 
Table 1 Frequency tables of chlamydia and gonorrhea cases and individual Indiana Network for Patient Care (INPC) records for Marion County between 2005 to 2014

\begin{tabular}{|c|c|c|c|c|c|c|c|c|c|c|}
\hline \multicolumn{11}{|c|}{ Number of reported chlamydia incidence cases for Marion County and the nine townships from INPC } \\
\hline Year & 2005 & 2006 & 2007 & 2008 & 2009 & 2010 & 2011 & 2012 & 2013 & 2014 \\
\hline Marion County & 4,281 & 4,205 & 4,410 & 4,754 & 5,123 & 5,075 & 5,258 & 5,301 & 4,793 & 4,425 \\
\hline Pike & 296 & 326 & 322 & 340 & 366 & 411 & 477 & 465 & 404 & 366 \\
\hline Washington & 425 & 388 & 383 & 448 & 473 & 495 & 518 & 584 & 427 & 428 \\
\hline Lawrence & 591 & 592 & 573 & 651 & 705 & 649 & 713 & 673 & 613 & 559 \\
\hline Wayne & 762 & 729 & 806 & 862 & 880 & 901 & 936 & 987 & 948 & 867 \\
\hline Central & 1,263 & 1,216 & 1,318 & 1,359 & 1,494 & 1,447 & 1,402 & 1,427 & 1,291 & 1,155 \\
\hline Warren & 568 & 568 & 602 & 641 & 670 & 656 & 705 & 709 & 648 & 590 \\
\hline Decatur & 85 & 76 & 73 & 66 & 113 & 107 & 104 & 86 & 74 & 108 \\
\hline Perry & 226 & 228 & 248 & 286 & 315 & 323 & 308 & 277 & 286 & 274 \\
\hline Franklin & 65 & 82 & 85 & 101 & 107 & 86 & 95 & 93 & 102 & 78 \\
\hline \multicolumn{11}{|c|}{ Number of reported gonorrhea incidence cases for Marion County and the nine townships from INPC } \\
\hline Year & 2005 & 2006 & 2007 & 2008 & 2009 & 2010 & 2011 & 2012 & 2013 & 2014 \\
\hline Marion County & 2692 & 2759 & 3038 & 3044 & 2898 & 2633 & 2504 & 2445 & 2225 & 2022 \\
\hline Pike & 167 & 192 & 192 & 203 & 178 & 181 & 215 & 201 & 170 & 167 \\
\hline Washington & 265 & 242 & 265 & 294 & 270 & 257 & 244 & 241 & 195 & 184 \\
\hline Lawrence & 415 & 391 & 388 & 435 & 420 & 357 & 353 & 338 & 313 & 261 \\
\hline Wayne & 413 & 443 & 501 & 515 & 464 & 454 & 405 & 414 & 389 & 352 \\
\hline Central & 849 & 889 & 1028 & 946 & 907 & 763 & 744 & 753 & 704 & 597 \\
\hline Warren & 374 & 377 & 428 & 406 & 438 & 364 & 357 & 334 & 296 & 316 \\
\hline Decatur & 53 & 46 & 42 & 40 & 45 & 44 & 37 & 35 & 24 & 26 \\
\hline Perry & 121 & 129 & 143 & 149 & 126 & 168 & 116 & 97 & 113 & 99 \\
\hline Franklin & 35 & 50 & 51 & 56 & 50 & 45 & 33 & 32 & 21 & 20 \\
\hline \multicolumn{11}{|c|}{ Population of individuals with a unique record in the INPC electronic health record system for Marion County and each township } \\
\hline Year & 2005 & 2006 & 2007 & 2008 & 2009 & 2010 & 2011 & 2012 & 2013 & 2014 \\
\hline Marion County & 555,034 & 589,968 & 635,742 & 654,213 & 710,340 & 721,201 & 695,418 & 676,883 & 642,296 & 548,170 \\
\hline Pike & 47,086 & 45,306 & 47,956 & 49,543 & 55,874 & 57,932 & 55,451 & 54,045 & 51,474 & 43,897 \\
\hline Washington & 70,592 & 68,544 & 73,883 & 76,771 & 85,399 & 89,273 & 86,270 & 85,179 & 78,027 & 63,435 \\
\hline
\end{tabular}




\begin{tabular}{|l|l|l|l|l|l|l|l|l|l|l|l|l|l|} 
Lawrence & 70,367 & 74,544 & 80,721 & 83,705 & 90,581 & 92,742 & 89,817 & 87,683 & 80,315 & 67,129 & 15 & 105 \\
Wayne & 88,266 & 89,164 & 97,508 & 101,466 & 110,280 & 112,447 & 107,435 & 102,831 & 100,661 & 88,338 \\
Central & 113,185 & 118,274 & 123,252 & 123,590 & 135,748 & 136,688 & 131,614 & 125,031 & 120,082 & 106,029 \\
Warren & 70,066 & 74,364 & 79,144 & 81,621 & 87,825 & 88,146 & 84,537 & 82,314 & 77,682 & 67,551 \\
Decatur & 17,414 & 20,180 & 22,206 & 23,012 & 24,082 & 24,021 & 23,011 & 23,038 & 22,143 & 19,560 \\
Perry & 54,226 & 68,367 & 75,488 & 78,280 & 82,581 & 82,282 & 80,225 & 79,938 & 77,298 & 64,435 \\
Franklin & 23,832 & 31,225 & 35,584 & 36,225 & 37,970 & 37,670 & 37,058 & 36,824 & 34,614 & 27,796 \\
\hline
\end{tabular}

Table 2 Descriptive statistics for chlamydia and gonorrhea rates for Marion County between 2005 to $2014^{\mathrm{a}, \mathrm{b}}$

\begin{tabular}{|c|c|c|c|c|c|c|c|c|c|c|}
\hline \multicolumn{11}{|c|}{ Range of lowest to highest chlamydia rates for Marion County and census tracts within each township } \\
\hline Year & 2005 & 2006 & 2007 & 2008 & 2009 & 2010 & 2011 & 2012 & 2013 & 2014 \\
\hline Marion County & $0.0-24.8$ & $0.0-22.3$ & $0.0-24.2$ & $0.0-26.4$ & $0.0-25.1$ & $0.0-20.5$ & $0.0-23.3$ & $0.0-26.9$ & $0.0-26.3$ & $0.0-22.7$ \\
\hline Pike & $0.6-12.4$ & $0.6-15.5$ & $1.0-13.2$ & $1.5-13.6$ & $0.9-12.2$ & $1.8-12.8$ & $1.4-18.0$ & $1.8-16.0$ & $1.0-14.8$ & $1.3-16.1$ \\
\hline Washington & $0.0-17.5$ & $0.0-18.9$ & $0.0-15.9$ & $0.0-18.7$ & $0.0-19.3$ & $0.0-18.9$ & $0.0-20.7$ & $0.0-25.6$ & $0.0-22.1$ & $0.0-22.5$ \\
\hline Lawrence & $0.0-19.3$ & $0.4-19.7$ & $0.0-18.7$ & $0.3-21.0$ & $0.0-25.1$ & $0.4-19.7$ & $0.3-18.8$ & $0.8-18.7$ & $0.0-16.8$ & $0.6-20.1$ \\
\hline Wayne & $1.9-18.0$ & $0.0-18.4$ & $1.7-17.5$ & $2.3-18.1$ & $0.8-14.0$ & $2.1-15.7$ & $0.8-17.3$ & $3.5-19.8$ & $3.2-17.0$ & $2.3-18.0$ \\
\hline Central & $2.2-24.8$ & $1.5-22.3$ & $2.4-24.2$ & $2.3-26.4$ & $3.3-24.9$ & $2.5-20.5$ & $4.0-23.3$ & $1.9-26.9$ & $2.5-26.3$ & $2.7-22.7$ \\
\hline Warren & $1.0-19.2$ & $0.0-19.2$ & $0.0-18.9$ & $0.8-19.4$ & $1.6-23.1$ & $2.7-17.1$ & $3.1-17.0$ & $3.1-16.9$ & $3.4-16.7$ & $2.2-19.0$ \\
\hline Decatur & $2.6-7.0$ & $2.2-4.7$ & $1.3-6.8$ & $0.8-4.2$ & $2.5-6.7$ & $2.8-6.6$ & $4.5-4.6$ & $0.7-7.0$ & $1.3-6.2$ & $4.7-6.7$ \\
\hline Perry & $0.6-10.8$ & $0.0-9.8$ & $0.5-8.9$ & $0.7-8.9$ & $1.1-8.6$ & $0.5-11.6$ & $0.0-8.5$ & $1.0-7.4$ & $1.2-8.0$ & $1.3-6.6$ \\
\hline Franklin & $0.8-4.0$ & $1.1-5.7$ & $0.0-4.9$ & $0.8-5.1$ & $1.6-4.3$ & $1.3-3.2$ & $1.6-4.0$ & $1.3-4.1$ & $1.4-4.8$ & $2.0-4.1$ \\
\hline \multicolumn{11}{|c|}{ Range of lowest to highest gonorrhea rates for Marion County and census tracts within each township } \\
\hline Year & 2005 & 2006 & 2007 & 2008 & 2009 & 2010 & 2011 & 2012 & 2013 & 2014 \\
\hline Marion County & $0.0-16.1$ & $0.0-16.8$ & $0.0-19.6$ & $0.0-16.1$ & $0.0-16.7$ & $0.0-13.8$ & $0.0-16.2$ & $0.0-17.6$ & $0.0-15.6$ & $0.0-14.7$ \\
\hline Pike & $0.0-8.0$ & $0.0-12.2$ & $1.0-8.4$ & $1.0-9.0$ & $0.0-5.4$ & $0.0-7.1$ & $0.0-8.4$ & $0.0-8.2$ & $0.5-6.0$ & $0.3-7.4$ \\
\hline Washington & $0.0-13.8$ & $0.0-14.9$ & $0.0-10.2$ & $0.0-14.0$ & $0.0-12.3$ & $0.0-11.8$ & $0.0-11.9$ & $0.0-11.1$ & $0.0-11.6$ & $0.0-10.9$ \\
\hline Lawrence & $0.0-15.5$ & $0.0-14.7$ & $0.0-13.7$ & $0.0-15.8$ & $0.0-15.5$ & $0.0-13.8$ & $0.0-10.6$ & $0.0-11.0$ & $0.0-8.7$ & $0.0-12.5$ \\
\hline Wayne & $0.0-14.1$ & $1.0-13.9$ & $0.8-13.6$ & $0.8-13.0$ & $0.4-9.4$ & $0.6-9.2$ & $0.0-9.2$ & $0.0-10.8$ & $0.0-8.9$ & $0.0-9.5$ \\
\hline Central & $1.1-15.6$ & $0.3-16.8$ & $2.1-19.6$ & $0.9-16.1$ & $1.5-16.7$ & $0.9-12.7$ & $1.2-16.2$ & $0.0-17.6$ & $1.0-15.6$ & $0.0-14.7$ \\
\hline Warren & $0.0-16.1$ & $0.9-14.5$ & $0.0-13.6$ & $0.0-13.8$ & $1.2-15.2$ & $0.4-10.7$ & $0.0-8.3$ & $0.0-10.7$ & $0.0-9.7$ & $0.6-10.5$ \\
\hline Decatur & $1.6-5.7$ & $0.4-4.1$ & $0.4-3.8$ & $0.9-3.1$ & $0.3-2.8$ & $1.1-3.0$ & $0.4-2.7$ & $0.0-3.1$ & $0.5-1.9$ & $0.7-2.7$ \\
\hline
\end{tabular}




\begin{tabular}{|c|c|c|c|c|c|c|c|c|c|c|}
\hline Perry & $0.5-5.6$ & $0.0-4.1$ & $0.0-4.1$ & $0.2-5.8$ & $0.0-5.1$ & $0.0-6.0$ & $0.0-3.6$ & $0.0-3.4$ & $0.0-3.6$ & $0.0-3.3$ \\
\hline Franklin & $0.0-4.0$ & $0.8-3.4$ & $0.0-3.2$ & $0.4-3.7$ & $0.4-3.0$ & $0.5-1.9$ & $0.0-2.2$ & $0.0-2.1$ & $0.0-1.2$ & $0.3-1.2$ \\
\hline \multicolumn{11}{|c|}{ Chlamydia rate for Marion County and the nine townships } \\
\hline Year & 2005 & 2006 & 2007 & 2008 & 2009 & 2010 & 2011 & 2012 & 2013 & 2014 \\
\hline Marion County & 7.71 & 7.13 & 6.94 & 7.27 & 7.21 & 7.04 & 7.56 & 7.83 & 7.46 & 8.07 \\
\hline Pike & 6.29 & 7.20 & 6.71 & 6.86 & 6.55 & 7.09 & 8.60 & 8.60 & 7.85 & 8.34 \\
\hline Washington & 6.02 & 5.66 & 5.18 & 5.84 & 5.54 & 5.54 & 6.00 & 6.86 & 5.47 & 6.75 \\
\hline Lawrence & 8.40 & 7.94 & 7.10 & 7.78 & 7.78 & 7.00 & 7.94 & 7.68 & 7.63 & 8.33 \\
\hline Wayne & 8.63 & 8.18 & 8.27 & 8.50 & 7.98 & 8.01 & 8.71 & 9.60 & 9.42 & 9.81 \\
\hline Central & 11.16 & 10.28 & 10.69 & 11.00 & 11.01 & 10.59 & 10.65 & 11.41 & 10.75 & 10.89 \\
\hline Warren & 8.11 & 7.64 & 7.61 & 7.85 & 7.63 & 7.44 & 8.34 & 8.61 & 8.34 & 8.73 \\
\hline Decatur & 4.88 & 3.77 & 3.29 & 2.87 & 4.69 & 4.45 & 4.52 & 3.73 & 3.34 & 5.52 \\
\hline Perry & 4.17 & 3.33 & 3.29 & 3.65 & 3.81 & 3.93 & 3.84 & 3.47 & 3.70 & 4.25 \\
\hline Franklin & 2.73 & 2.63 & 2.39 & 2.79 & 2.82 & 2.28 & 2.56 & 2.53 & 2.95 & 2.81 \\
\hline \multicolumn{11}{|c|}{ Gonorrhea rate for Marion County and the nine townships } \\
\hline Year & 2005 & 2006 & 2007 & 2008 & 2009 & 2010 & 2011 & 2012 & 2013 & 2014 \\
\hline Marion County & 4.85 & 4.68 & 4.78 & 4.65 & 4.08 & 3.65 & 3.60 & 3.61 & 3.46 & 3.69 \\
\hline Pike & 3.55 & 4.24 & 4.00 & 4.10 & 3.19 & 3.12 & 3.88 & 3.72 & 3.30 & 3.80 \\
\hline Washington & 3.75 & 3.53 & 3.59 & 3.83 & 3.16 & 2.88 & 2.83 & 2.83 & 2.50 & 2.90 \\
\hline Lawrence & 5.90 & 5.25 & 4.81 & 5.20 & 4.64 & 3.85 & 3.93 & 3.85 & 3.90 & 3.89 \\
\hline Wayne & 4.68 & 4.97 & 5.14 & 5.08 & 4.21 & 4.04 & 3.77 & 4.03 & 3.86 & 3.98 \\
\hline Central & 7.50 & 7.52 & 8.34 & 7.65 & 6.68 & 5.58 & 5.65 & 6.02 & 5.86 & 5.63 \\
\hline Warren & 5.34 & 5.07 & 5.41 & 4.97 & 4.99 & 4.13 & 4.22 & 4.06 & 3.81 & 4.68 \\
\hline Decatur & 3.04 & 2.28 & 1.89 & 1.74 & 1.87 & 1.83 & 1.61 & 1.52 & 1.08 & 1.33 \\
\hline Perry & 2.23 & 1.89 & 1.89 & 1.90 & 1.53 & 2.04 & 1.45 & 1.21 & 1.46 & 1.54 \\
\hline Franklin & 1.47 & 1.60 & 1.43 & 1.55 & 1.32 & 1.19 & 0.89 & 0.87 & 0.61 & 0.72 \\
\hline \multicolumn{11}{|c|}{ Standard deviation of chlamydia rates for Marion County and census tracts within each township } \\
\hline Year & 2005 & 2006 & 2007 & 2008 & 2009 & 2010 & 2011 & 2012 & 2013 & 2014 \\
\hline Marion County & 4.9 & 4.9 & 5.0 & 5.3 & 5.2 & 4.7 & 5.0 & 5.2 & 4.9 & 4.9 \\
\hline Pike & 3.9 & 3.9 & 3.2 & 3.5 & 3.1 & 3.4 & 4.1 & 4.3 & 4.0 & 3.7 \\
\hline Washington & 4.4 & 4.4 & 3.9 & 5.2 & 4.9 & 4.8 & 5.2 & 5.1 & 4.4 & 5.2 \\
\hline
\end{tabular}




\begin{tabular}{|c|c|c|c|c|c|c|c|c|c|c|}
\hline Lawrence & 5.8 & 6.1 & 5.9 & 6.6 & 7.1 & 5.5 & 5.9 & 6.0 & 5.4 & 5.7 \\
\hline Wayne & 4.4 & 4.2 & 4.3 & 4.0 & 3.6 & 3.4 & 3.9 & 4.2 & 3.7 & 4.3 \\
\hline Central & 4.4 & 4.2 & 4.8 & 4.9 & 4.8 & 4.0 & 4.7 & 4.9 & 4.5 & 4.2 \\
\hline Warren & 5.2 & 4.7 & 4.8 & 5.1 & 5.0 & 4.7 & 4.2 & 4.3 & 4.2 & 4.5 \\
\hline Decatur & 1.9 & 1.1 & 2.3 & 1.3 & 1.7 & 1.4 & 0.0 & 2.4 & 1.9 & 0.9 \\
\hline Perry & 2.3 & 2.3 & 2.3 & 2.4 & 1.8 & 2.8 & 2.1 & 1.9 & 2.1 & 1.5 \\
\hline Franklin & 1.1 & 1.7 & 1.7 & 1.6 & 1.1 & 0.7 & 0.7 & 1.0 & 1.2 & 0.6 \\
\hline \multicolumn{11}{|c|}{ Standard deviation of gonorrhea rates for Marion County and census tracts within each township } \\
\hline Year & 2005 & 2006 & 2007 & 2008 & 2009 & 2010 & 2011 & 2012 & 2013 & 2014 \\
\hline Marion County & 3.8 & 3.8 & 4.1 & 3.9 & 3.6 & 2.9 & 3.1 & 3.2 & 3.0 & 3.0 \\
\hline Pike & 2.3 & 3.1 & 2.0 & 2.3 & 1.7 & 1.9 & 2.2 & 2.5 & 1.7 & 1.9 \\
\hline Washington & 3.2 & 3.4 & 3.3 & 3.8 & 3.2 & 2.9 & 3.1 & 2.8 & 2.9 & 2.6 \\
\hline Lawrence & 4.8 & 4.5 & 4.3 & 4.5 & 4.5 & 3.4 & 3.5 & 3.5 & 2.8 & 3.3 \\
\hline Wayne & 3.4 & 3.1 & 3.2 & 2.8 & 2.3 & 1.7 & 2.1 & 2.0 & 2.2 & 2.5 \\
\hline Central & 3.5 & 3.5 & 4.1 & 3.6 & 3.7 & 2.9 & 3.5 & 3.4 & 3.0 & 3.0 \\
\hline Warren & 3.7 & 3.5 & 3.3 & 3.9 & 3.5 & 3.0 & 2.4 & 2.8 & 2.7 & 2.8 \\
\hline Decatur & 1.7 & 1.6 & 1.3 & 0.9 & 1.0 & 0.8 & 0.9 & 1.0 & 0.6 & 0.8 \\
\hline Perry & 1.4 & 1.3 & 1.3 & 1.6 & 1.1 & 1.4 & 0.9 & 0.9 & 1.2 & 1.0 \\
\hline Franklin & 1.5 & 0.9 & 1.0 & 1.0 & 0.8 & 0.5 & 0.7 & 0.7 & 0.4 & 0.4 \\
\hline
\end{tabular}

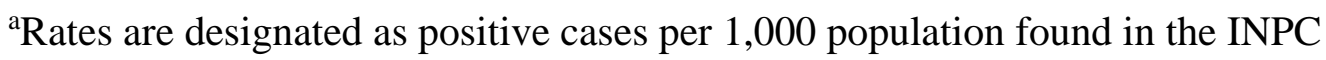

${ }^{\mathrm{b}}$ The denominator to calculate rates is derived from the population of individuals with a record in the INPC electronic health record system for each township. 
2005

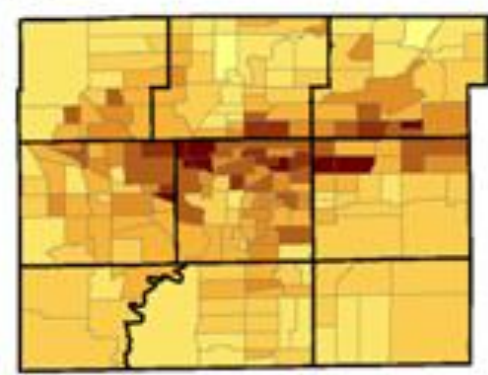

2007

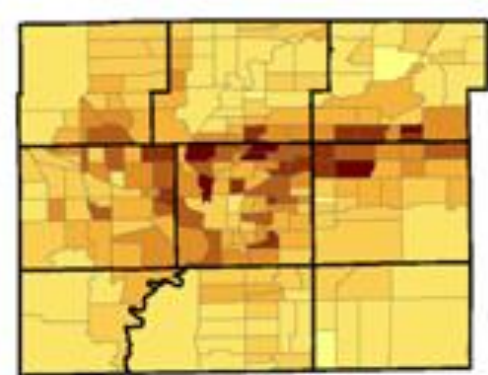

2006

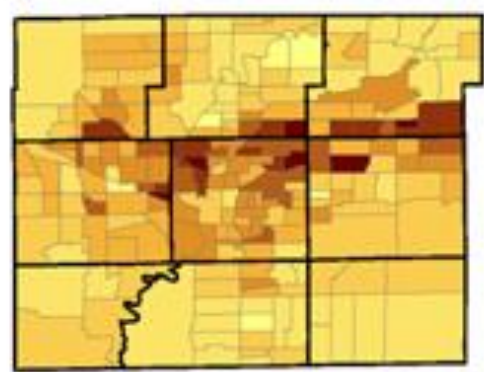

2008

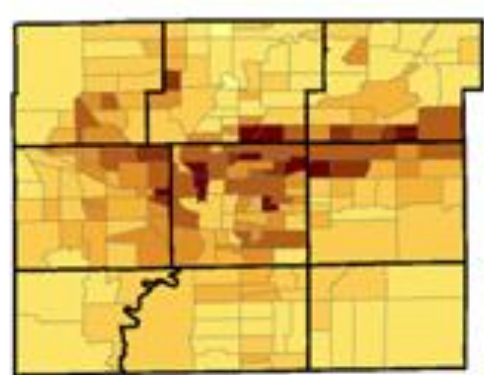

2009

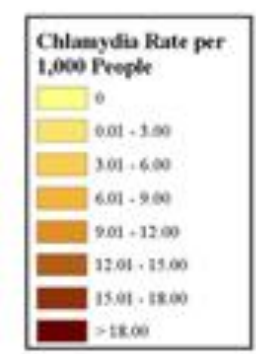

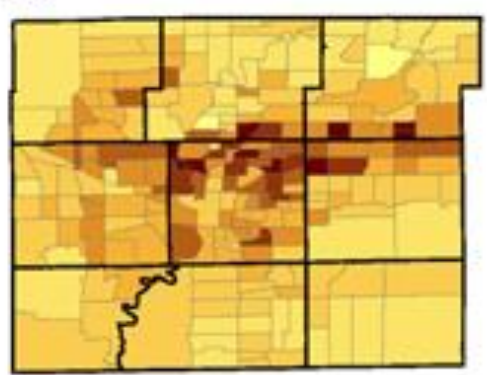

2010

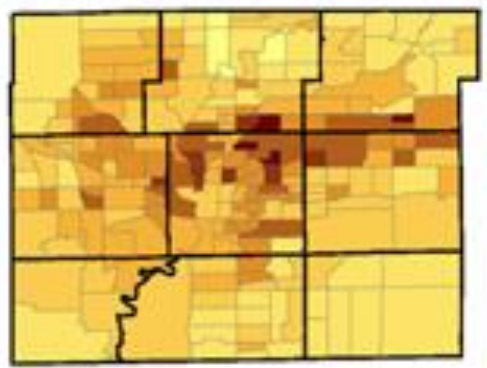

2012
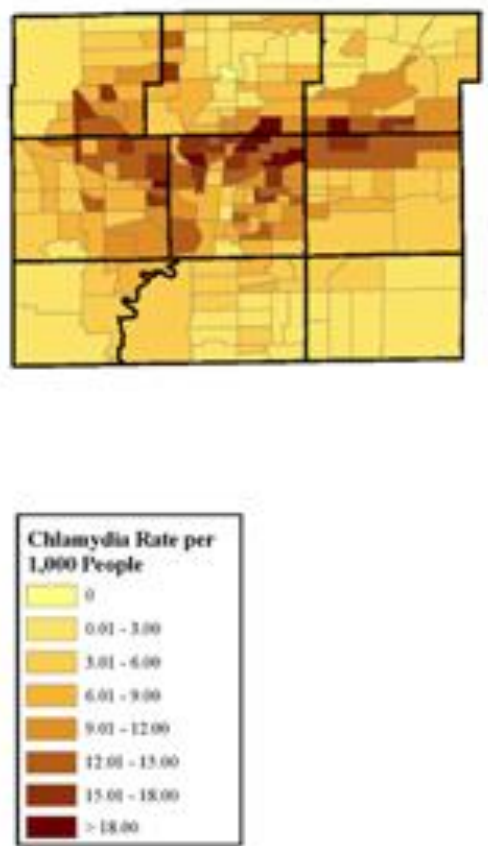

2011

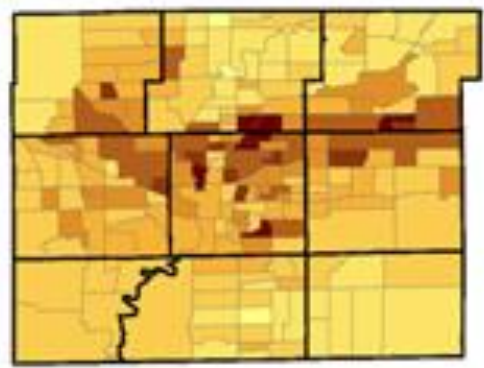

2013

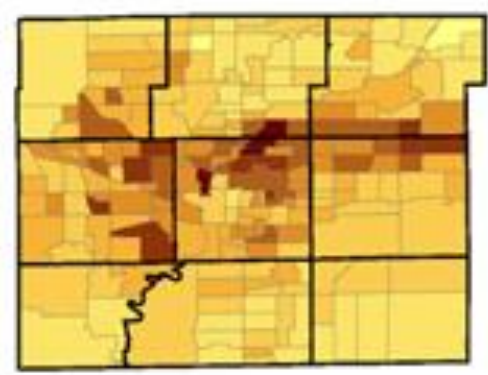

2014

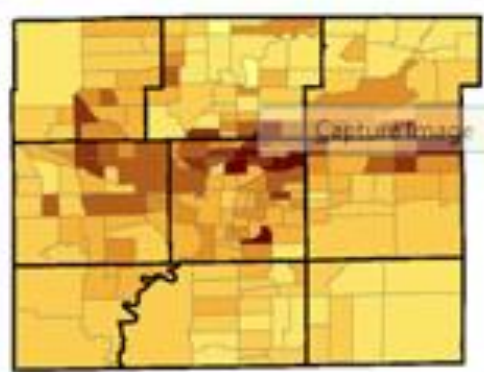

Figure 3 Spatial visualization of chlamydia rates per 1,000 people for each census tract in Marion County from 2005-2014 
2005

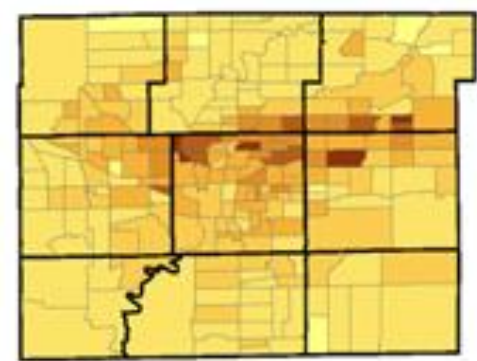

2007
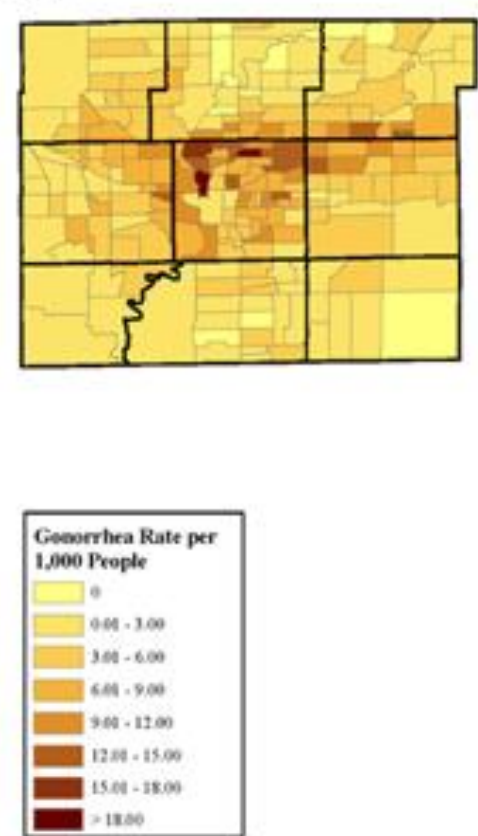

2006

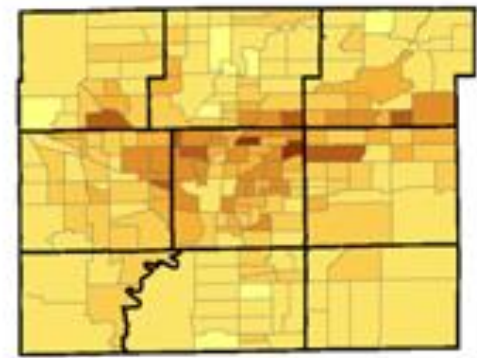

2008

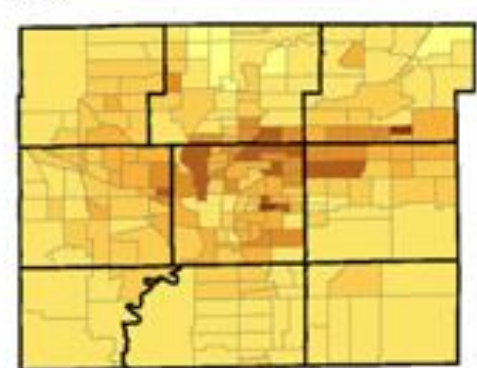

2009

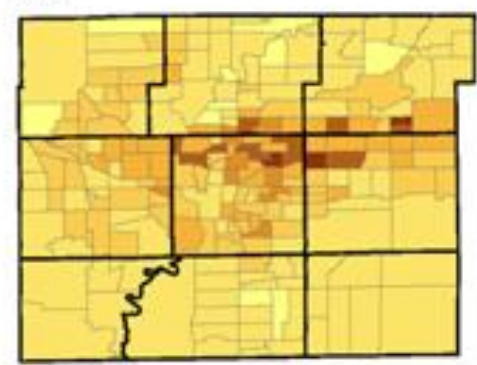

2010

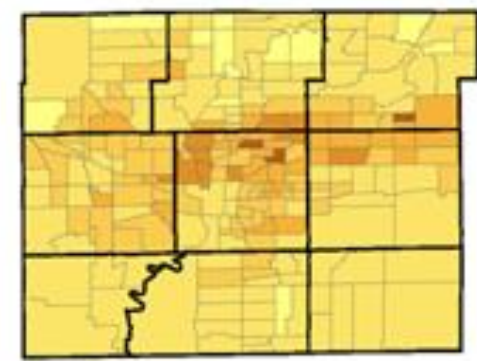

2012

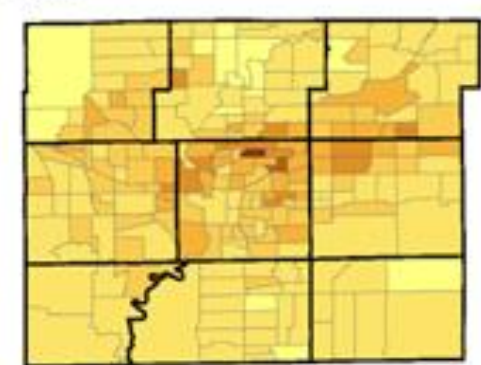

\begin{tabular}{|c|c|}
\hline \multicolumn{2}{|c|}{$\begin{array}{l}\text { Gonorrthea Rate per } \\
\text { 1,000 Prople }\end{array}$} \\
\hline & \\
\hline & Den - 300 \\
\hline & $3 e n-6000$ \\
\hline & $601-300$ \\
\hline & $964=1200$ \\
\hline & $12=1500$ \\
\hline & $180,-18000$ \\
\hline
\end{tabular}

2011

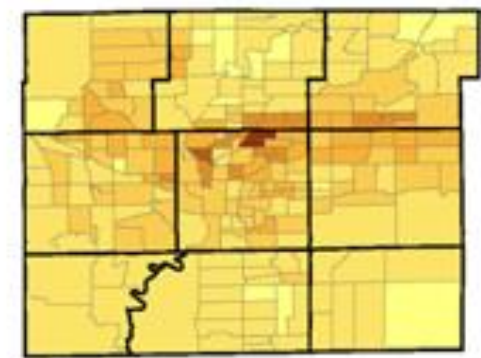

2013

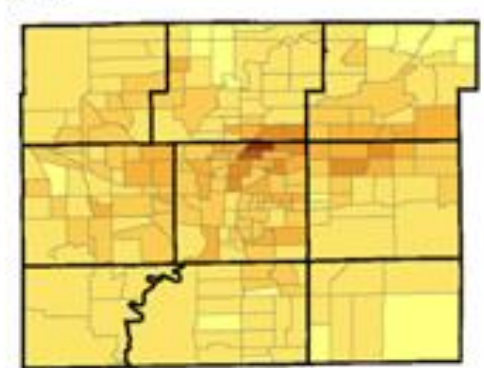

2014

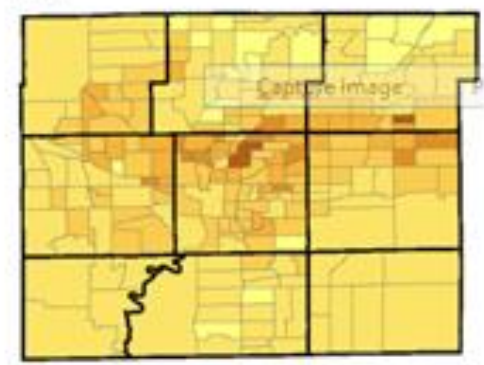

Figure 4 Spatial visualization of gonorrhea rates per 1,000 people for each census tract in Marion County from 2005-2014 
2005

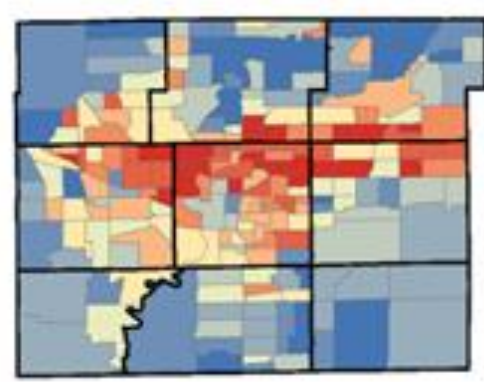

2007

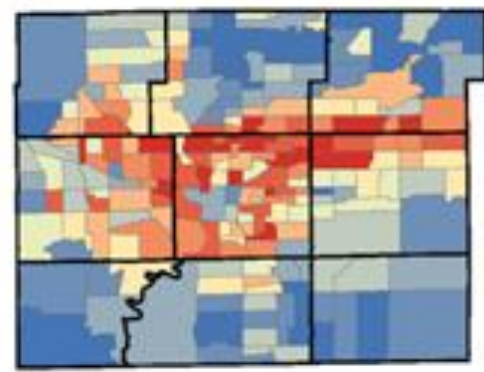

Cerisus Tract Decile Grouping by Chamydia Rate is Decile deses rove

- and Derile

Mod Dedil
ation

so Dech

atocia

at beilt

7 isencte

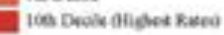

2006

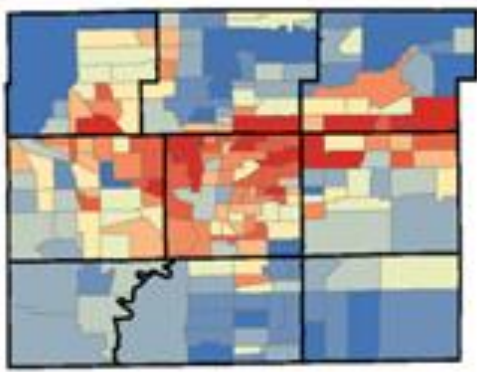

2008

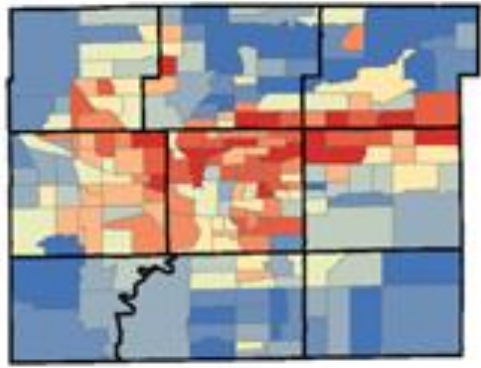

2009

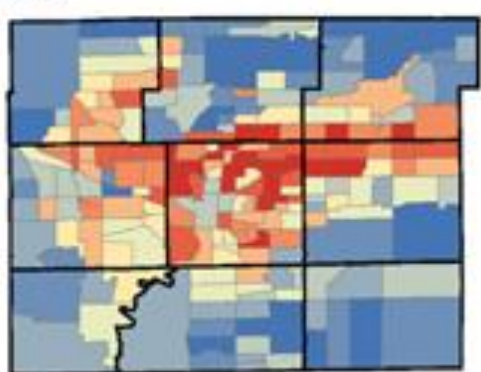

2010

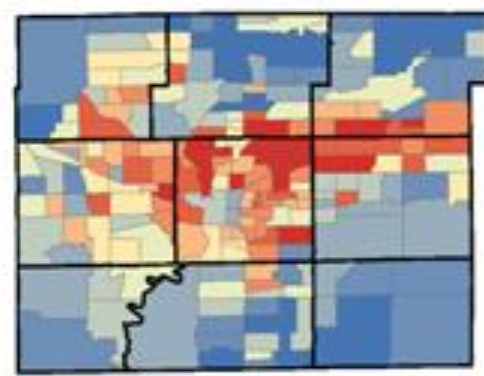

2012

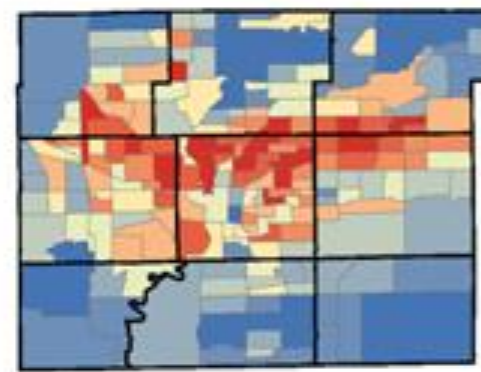

Census Trast Decile Greopire

by Chbanydia Rate

Wis Docile

Ind Decile

moscles

us pirite

Th Descic

mos Dock

se Denck

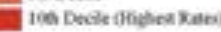

2011

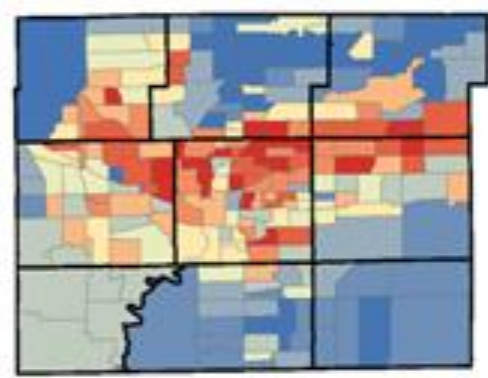

2013

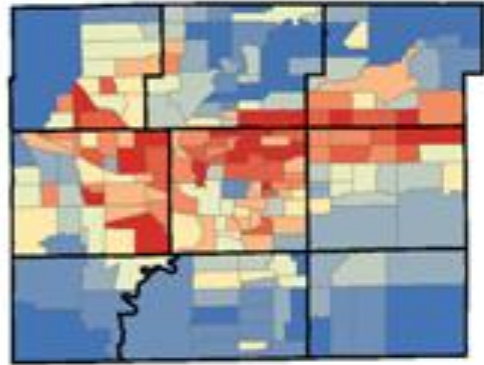

2014

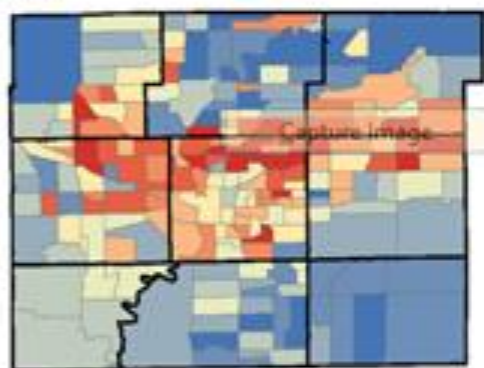

Figure 5 Spatial visualization of chlamydia decile grouping for each census tract in Marion County from 2005-2014 
2005

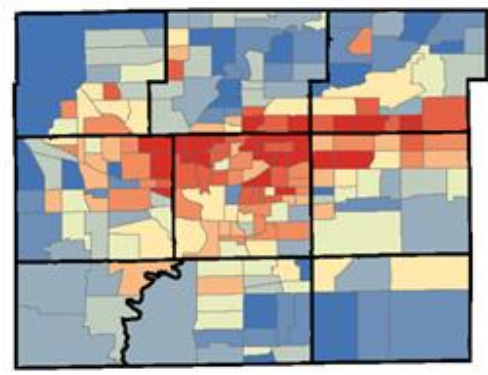

2007

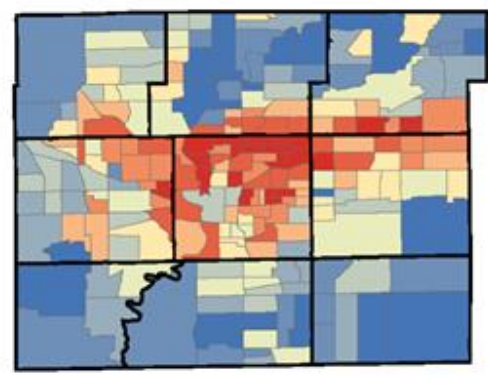

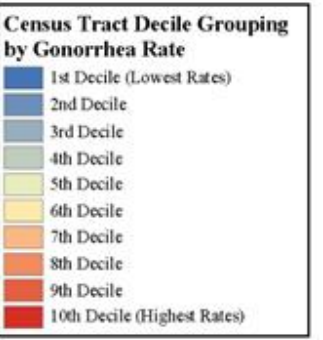

2006

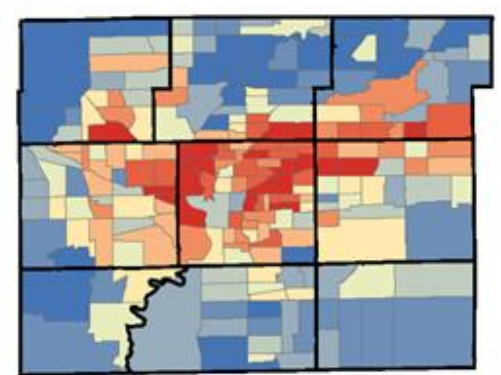

2008

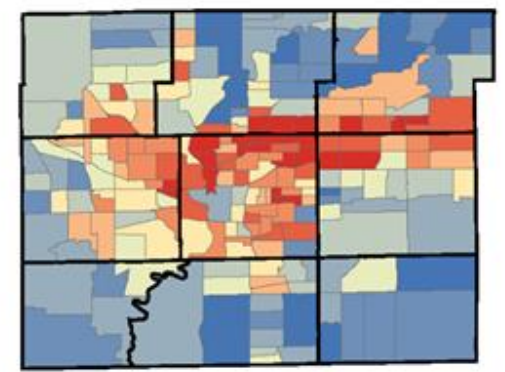

2009

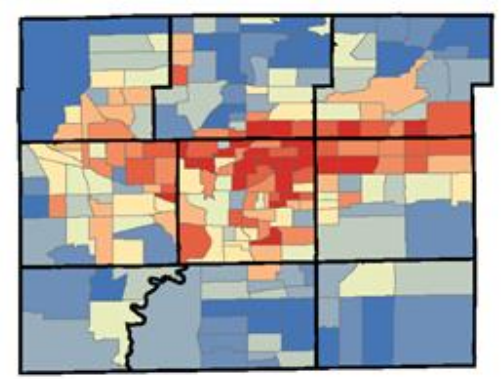

2010

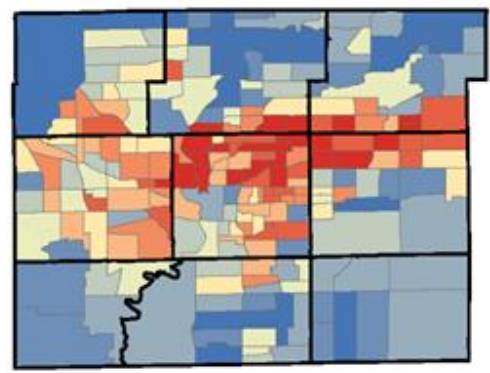

2012

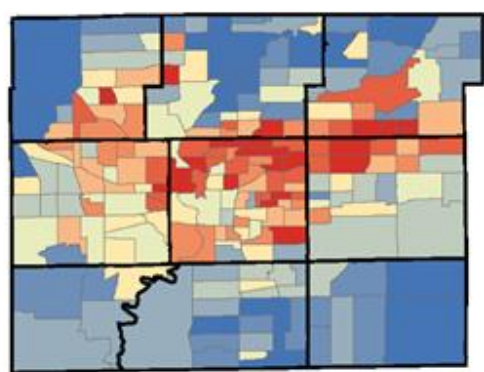

Census Tract Decile Grouping by Gonorrhea Rate Is Decile (Lowes Rates)

2nd Decile

3rd Decile
4th Decile

4h Decile
Sth Decile

6th Decile

7 th Decile

sth Decile

1 oet Decile (Highes Rates)
2011

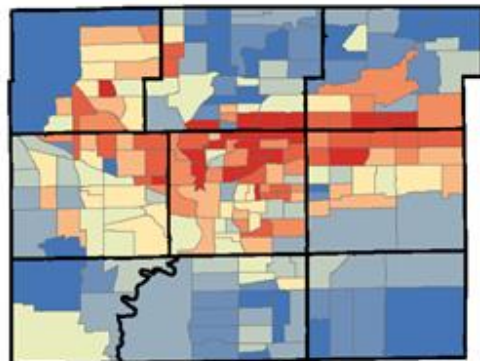

2013

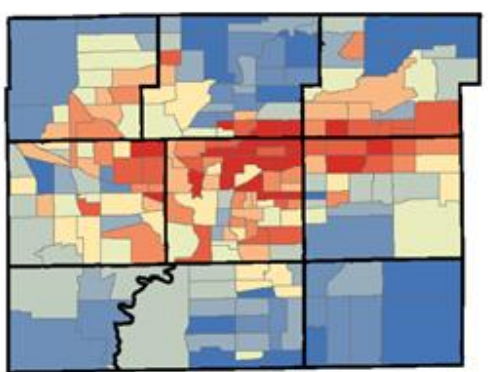

2014

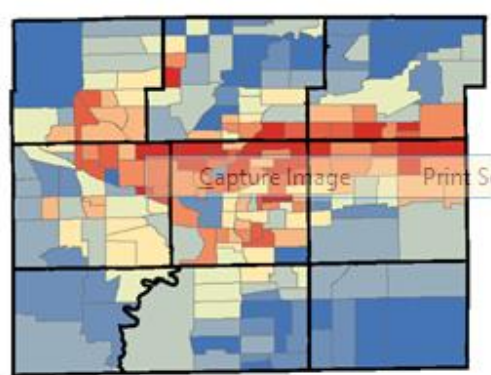

Figure 6 Spatial visualization of gonorrhea decile grouping for each census tract in Marion County from 2005-2014 
To investigate the magnitude of inequality of disease rates by individual townships, Table 4 represents a colored display of the Gini coefficients for chlamydia and gonorrhea. Gini coefficients that are more distributed evenly with lesser inequality were shown in darker green colors while Gini coefficients that have greater inequality were shown in darker red colors. The highest Gini coefficients for both diseases were found consistently in Washington and Lawrence Townships while Central Township had one of the lowest Gini coefficient values during the ten-year period. Although there were periodic spikes in inequality for Decatur and Franklin Townships, both locations had low values in the Gini coefficients for specific years such as 2011 and 2014 for chlamydia and 2010 for gonorrhea. Additionally, Decatur and Franklin were the two townships that experienced more fluctuation in Gini coefficients over time compared to other townships. This is exhibited between 2006 to 2007, 2011 to 2012, and 2013 to 2014 for chlamydia for Decatur Township and in the time period between 2005 to 2006 and 2010 to 2011 for gonorrhea for Franklin Township. Overall throughout the nine townships, the individual Gini coefficients for gonorrhea were mostly higher than those for chlamydia.

Finally, to determine if there was a difference in the average Gini coefficient within each year and within each township, a one-way ANOVA test was calculated for both diseases. For chlamydia, the average Gini coefficients for each township in the ten-year period were as follows: Pike = 0.312 , Washington $=0.471$, Lawrence $=0.476$, Wayne $=0.269$, Central $=0.231$, Warren $=0.323$, Decatur $=0.249$, Perry $=0.322$, and Franklin $=0.254$. When the Gini coefficients were compared by year, the overall results were not statistically significant $(F(9,80)=0.92$, root mean squared $=$ $0.106, p=0.5152)$. However, when the Gini coefficients were compared between the nine townships or geographical locations, there was an overall statistically significant result $(F(8,81)$ $=21.40$, root mean squared $=0.063, p<0.0001$ ). A post-hoc multiple comparisons test with a Tukey adjustment was applied to determine which townships were different from each other in terms of the average Gini coefficient as shown in Table 5. The results showed that some townships especially Lawrence and Washington exhibited differences in average Gini coefficients compared to all other townships $(p<0.0001)$. Consequently, Lawrence and Washington did not have significant differences in Gini coefficients when compared to each other. Other statistically significant differences in Gini coefficients were observed between Central and Warren Townships $(p=0.0228)$ and between Perry and Central Townships $(p=0.0255)$.

For gonorrhea, the same multiple comparisons test was applied, and results are shown in Table 5. The average Gini coefficients for each township in the ten-year period were as follows: Pike $=$ 0.364 , Washington $=0.564$, Lawrence $=0.529$, Wayne $=0.330$, Central $=0.283$, Warren $=0.370$, Decatur $=0.366$, Perry $=0.406$, and Franklin $=0.395$. When the Gini coefficients were compared by year, there was no statistically significant result in the differences of the Gini coefficients $(F(9$, $80)=0.20$, root mean squared $=0.103, p=0.9940$ ). However, when the Gini coefficients were compared within the nine townships or geographical location, there was an overall statistically significant result $(F(8,81)=30.93$, root mean squared $=0.051, p<0.0001)$. Using the same posthoc multiple comparisons test, the township pairings that exhibited statistically significant findings for chlamydia were also statistically significant for gonorrhea. In addition, there were additional statistically significant township pairings that were not previously found in chlamydia including Central-Pike townships, Wayne-Perry townships, Central-Franklin townships, and CentralDecatur townships. 
Table 3 Summary statistics of chlamydia and gonorrhea decile grouping among Marion County census tracts from 2005 to $2014^{\mathrm{a}, \mathrm{b}, \mathbf{c}, \mathrm{d}}$

\begin{tabular}{|c|c|c|c|c|c|c|c|c|c|c|}
\hline \multicolumn{11}{|l|}{ Chlamydia } \\
\hline \multirow{2}{*}{$\begin{array}{l}\text { Year } \\
\text { Decile Group }\end{array}$} & \multicolumn{2}{|l|}{2005} & \multicolumn{2}{|l|}{2006} & \multicolumn{2}{|l|}{2007} & \multicolumn{2}{|l|}{2008} & \multicolumn{2}{|l|}{2009} \\
\hline & \% Рop & \% Cases & \% Рop & \% Cases & \% Рop & $\%$ Cases & \% Рop & \% Cases & \% Рop & $\%$ Cases \\
\hline $1^{\text {st }}$ Decile & 8.8 & 1.4 & 8.8 & 1.0 & 9.8 & 1.1 & 9.5 & 1.1 & 9.4 & 1.5 \\
\hline $2^{\text {nd }}$ Decile & 9.8 & 3.0 & 9.9 & 2.9 & 10.8 & 2.8 & 9.6 & 2.6 & 10.0 & 3.4 \\
\hline $3^{\text {rd }}$ Decile & 10.6 & 5.1 & 11.5 & 5.2 & 10.1 & 4.5 & 12.4 & 5.6 & 12.0 & 5.6 \\
\hline $4^{\text {th }}$ Decile & 9.9 & 6.4 & 11.4 & 7.1 & 11.2 & 7.1 & 11.7 & 7.4 & 9.9 & 6.0 \\
\hline $5^{\text {th }}$ Decile & 10.0 & 8.2 & 10.1 & 8.2 & 8.4 & 6.7 & 10.2 & 7.8 & 10.6 & 8.1 \\
\hline $6^{\text {th }}$ Decile & 10.6 & 10.2 & 10.3 & 10.7 & 10.5 & 10.5 & 8.7 & 8.6 & 9.5 & 9.3 \\
\hline $7^{\text {th }}$ Decile & 9.9 & 11.8 & 9.0 & 11.0 & 10.0 & 12.2 & 9.8 & 12.3 & 10.7 & 13.0 \\
\hline $8^{\text {th }}$ Decile & 10.6 & 15.4 & 8.7 & 12.4 & 10.5 & 15.5 & 8.9 & 13.7 & 10.4 & 15.4 \\
\hline $9^{\text {th }}$ Decile & 10.1 & 17.3 & 10.1 & 17.8 & 10.4 & 19.0 & 10.1 & 18.5 & 9.1 & 16.4 \\
\hline $10^{\text {th }}$ Decile & 9.6 & 21.2 & 10.2 & 23.7 & 8.3 & 20.6 & 9.2 & 22.4 & 8.3 & 21.3 \\
\hline Top Half & 50.8 & 75.9 & 48.3 & 75.6 & 49.7 & 77.8 & 46.7 & 75.5 & 48.0 & 75.4 \\
\hline $\begin{array}{l}\text { County Gini } \\
\text { Coefficient }\end{array}$ & \multicolumn{2}{|l|}{0.362} & \multicolumn{2}{|l|}{0.380} & \multicolumn{2}{|l|}{0.392} & \multicolumn{2}{|l|}{0.394} & \multicolumn{2}{|l|}{0.383} \\
\hline Year & \multicolumn{2}{|l|}{2010} & \multicolumn{2}{|l|}{2011} & \multicolumn{2}{|l|}{2012} & \multicolumn{2}{|l|}{2013} & \multicolumn{2}{|l|}{2014} \\
\hline Decile Group & \% Рop & $\%$ Cases & \% Pop & $\%$ Cases & $\%$ Pop & $\%$ Cases & \% Pop & $\%$ Cases & \% Рop & $\%$ Cases \\
\hline $1^{\text {st }}$ Decile & 10.0 & 1.5 & 8.9 & 1.4 & 9.7 & 1.5 & 9.7 & 1.6 & 8.1 & 1.5 \\
\hline $2^{\text {nd }}$ Decile & 11.0 & 3.7 & 11.8 & 4.2 & 11.2 & 3.7 & 12.5 & 4.3 & 9.8 & 3.4 \\
\hline $3^{\text {rd }}$ Decile & 11.1 & 5.4 & 11.2 & 5.6 & 12.2 & 5.8 & 9.6 & 4.8 & 11.2 & 5.7 \\
\hline $4^{\text {th }}$ Decile & 10.9 & 6.6 & 11.8 & 7.7 & 11.3 & 7.6 & 10.2 & 6.8 & 11.3 & 7.4 \\
\hline $5^{\text {th }}$ Decile & 9.6 & 8.1 & 8.3 & 6.8 & 9.0 & 7.8 & 10.2 & 6.8 & 10.0 & 8.1 \\
\hline
\end{tabular}




\begin{tabular}{|l|l|l|l|l|l|l|l|l|l|l|}
\hline $6^{\text {th }}$ Decile & 9.3 & 9.8 & 8.5 & 8.6 & 8.1 & 8.2 & 9.6 & 9.8 & 9.3 & 9.2 \\
\hline $7^{\text {th }}$ Decile & 9.5 & 12.1 & 10.4 & 12.3 & 10.6 & 12.9 & 9.2 & 11.4 & 9.7 & 11.3 \\
\hline $8^{\text {th }}$ Decile & 9.5 & 14.6 & 9.2 & 13.9 & 9.0 & 14.2 & 9.9 & 14.4 & 9.7 & 13.5 \\
\hline $9^{\text {th }}$ Decile & 10.4 & 18.6 & 11.6 & 20.3 & 9.2 & 16.4 & 9.8 & 17.6 & 10.5 & 17.5 \\
\hline $10^{\text {th }}$ Decile & 8.7 & 19.6 & 8.3 & 19.1 & 9.5 & 21.9 & 9.3 & 20.8 & 10.4 & 22.4 \\
\hline Top Half & 47.4 & 74.7 & 48.0 & 74.2 & 46.4 & 73.6 & 47.8 & 74.0 & 49.6 & 73.9 \\
\hline $\begin{array}{l}\text { County Gini } \\
\text { Coefficient }\end{array}$ & 0.365 & 0.361 & & 0.362 & & 0.356 & & 0.344 & \\
\hline
\end{tabular}

\section{Gonorrhea}

\begin{tabular}{|l|l|l|l|l|l|l|l|l|l|l|}
\hline Year & $\mathbf{2 0 0 5}$ & \multicolumn{2}{l}{$\mathbf{2 0 0 6}$} & $\mathbf{2 0 0 7}$ & \multicolumn{2}{l|}{$\mathbf{2 0 0 8}$} & \multicolumn{2}{l|}{ 2009 } \\
\hline Decile Group & $\mathbf{\%}$ Pop & \% Cases & \% Pop & \% Cases & \% Pop & \% Cases & \% Pop & \% Cases & \% Pop & \% Cases \\
\hline $1^{\text {st }}$ Decile & 8.3 & 0.8 & 8.9 & 0.6 & 9.5 & 0.5 & 8.9 & 0.7 & 9.5 & 0.6 \\
\hline $2^{\text {nd }}$ Decile & 8.8 & 2.0 & 10.8 & 2.6 & 10.4 & 2.3 & 10.6 & 2.3 & 9.6 & 2.2 \\
\hline $3^{\text {rd }}$ Decile & 11.5 & 4.2 & 10.7 & 3.8 & 11 & 4.3 & 11.7 & 4.2 & 11.5 & 4.4 \\
\hline $4^{\text {th }}$ Decile & 9.7 & 5.2 & 9.4 & 5.0 & 10.4 & 5.8 & 12.0 & 6.7 & 11.2 & 5.8 \\
\hline $5^{\text {th }}$ Decile & 10.7 & 7.1 & 10.0 & 7.1 & 11.6 & 9.0 & 10.4 & 7.7 & 10.4 & 7.1 \\
\hline $6^{\text {th }}$ Decile & 10.3 & 9.2 & 10.9 & 9.8 & 9.0 & 8.5 & 7.9 & 7.1 & 8.6 & 8.0 \\
\hline $7^{\text {th }}$ Decile & 10.7 & 13.0 & 10.9 & 12.7 & 10.9 & 13.3 & 10.5 & 12.8 & 11.6 & 13.4 \\
\hline $8^{\text {th }}$ Decile & 10.5 & 15.8 & 10.1 & 15.8 & 10.4 & 16.6 & 9.6 & 15.7 & 9.1 & 13.7 \\
\hline $9^{\text {th }}$ Decile & 9.5 & 17.0 & 9.0 & 18.3 & 8.9 & 17.7 & 10.2 & 20.0 & 9.7 & 18.3 \\
\hline $10^{\text {th }}$ Decile & 10.0 & 25.7 & 9.1 & 24.3 & 7.8 & 22.0 & 8.3 & 22.8 & 8.8 & 26.5 \\
\hline Top Half & 51.0 & 80.7 & 50.0 & 80.9 & 47.0 & 78.1 & 46.5 & 78.4 & 47.8 & 79.9 \\
\hline $\begin{array}{l}\text { County Gini } \\
\text { Coefficient }\end{array}$ & 0.431 & & 0.440 & & 0.442 & & 0.441 & & 0.454 \\
\hline
\end{tabular}




\begin{tabular}{|l|l|l|l|l|l|l|l|l|l|l|}
\hline Year & $\mathbf{2 0 1 0}$ & \multicolumn{2}{l}{$\mathbf{2 0 1 1}$} & $\mathbf{2 0 1 2}$ & \multicolumn{2}{l|}{$\mathbf{2 0 1 3}$} & \multicolumn{2}{l|}{ 2014 } \\
\hline Decile Group & \% Pop & \% Cases & \% Pop & \% Cases & \% Pop & \% Cases & \% Pop & \% Cases & \% Pop & \% Cases \\
\hline $1^{\text {st }}$ Decile & 9.5 & 0.6 & 7.9 & 0.4 & 8.9 & 0.4 & 8.7 & 0.3 & 8.7 & 0.3 \\
\hline $2^{\text {nd }}$ Decile & 10.1 & 2.7 & 10.5 & 2.3 & 10.5 & 1.9 & 11.0 & 2.0 & 9.2 & 1.8 \\
\hline $3^{\text {rd }}$ Decile & 13.1 & 6.0 & 13.3 & 5.1 & 12.6 & 4.6 & 10.4 & 3.6 & 10.8 & 3.4 \\
\hline $4^{\text {th }}$ Decile & 10.1 & 6.2 & 10.4 & 5.9 & 11.7 & 6.7 & 12.4 & 6.5 & 11.6 & 5.7 \\
\hline $5^{\text {th }}$ Decile & 9.1 & 7.0 & 10.0 & 7.5 & 8.6 & 6.4 & 9.4 & 7.0 & 9.2 & 6.7 \\
\hline $6^{\text {th }}$ Decile & 10.3 & 9.8 & 9.8 & 9.3 & 10.4 & 9.7 & 10 & 9.9 & 10.6 & 10.2 \\
\hline $7^{\text {th }}$ Decile & 10.1 & 11.7 & 10.2 & 12.1 & 9.3 & 11.2 & 9.8 & 12 & 10.5 & 13.1 \\
\hline $8^{\text {th }}$ Decile & 8.1 & 12 & 9.5 & 14.6 & 9.6 & 14.6 & 10.1 & 16.3 & 9.2 & 13.6 \\
\hline $9^{\text {th }}$ Decile & 10.9 & 20.6 & 10.3 & 19.6 & 9.3 & 18.7 & 9.9 & 19.4 & 9.9 & 18.4 \\
\hline $10^{\text {th }}$ Decile & 8.8 & 23.4 & 8.2 & 23.3 & 9.1 & 25.9 & 8.3 & 23.1 & 10.1 & 26.6 \\
\hline Top Half & 48.2 & 77.5 & 48.0 & 78.9 & 47.7 & 80.1 & 48.1 & 80.7 & 50.3 & 81.9 \\
\hline $\begin{array}{l}\text { County Gini } \\
\text { Coefficient }\end{array}$ & 0.417 & & 0.445 & & 0.452 & & 0.452 & & 0.445 \\
\hline
\end{tabular}

${ }^{\mathrm{a}} 1^{\text {st }}$ decile grouping represents the $10 \%$ of census tracts with the lowest disease rates. ${ }^{\mathrm{b}} 10^{\text {th }}$ decile grouping represents the $10 \%$ of census tracts with the highest disease rates. ${ }^{c} \%$ Pop is percent population which is derived from the population of individuals with a record in

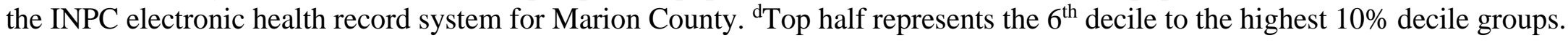


Table 4 Gini coefficients of chlamydia and gonorrhea rate inequality among townships between 2005 to $2014^{\mathrm{a}}$

\begin{tabular}{|c|c|c|c|c|c|c|c|c|c|c|}
\hline \multicolumn{11}{|l|}{ Chlamydia } \\
\hline \multirow{2}{*}{ Township } & \multicolumn{10}{|l|}{ Year } \\
\hline & 2005 & 2006 & 2007 & 2008 & 2009 & 2010 & 2011 & 2012 & 2013 & 2014 \\
\hline Pike & 0.377 & 0.343 & 0.306 & 0.315 & 0.293 & 0.299 & 0.275 & 0.312 & 0.319 & 0.274 \\
\hline Washington & 0.434 & 0.466 & 0.453 & 0.517 & 0.501 & 0.496 & 0.498 & 0.428 & 0.44 & 0.471 \\
\hline Lawrence & 0.444 & 0.489 & 0.511 & 0.516 & 0.518 & 0.470 & 0.451 & 0.464 & 0.452 & 0.452 \\
\hline Wayne & 0.289 & 0.292 & 0.307 & 0.270 & 0.272 & 0.245 & 0.265 & 0.251 & 0.227 & 0.261 \\
\hline Central & 0.218 & 0.221 & 0.246 & 0.244 & 0.239 & 0.211 & 0.240 & 0.240 & 0.228 & 0.216 \\
\hline Warren & 0.360 & 0.342 & 0.344 & 0.356 & 0.339 & 0.339 & 0.280 & 0.277 & 0.287 & 0.298 \\
\hline Decatur & 0.256 & 0.189 & 0.430 & 0.310 & 0.243 & 0.198 & 0.005 & 0.415 & 0.333 & 0.101 \\
\hline Perry & 0.295 & 0.355 & 0.379 & 0.361 & 0.277 & 0.391 & 0.307 & 0.314 & 0.308 & 0.220 \\
\hline Franklin & 0.220 & 0.369 & 0.430 & 0.360 & 0.22 & 0.181 & 0.144 & 0.232 & 0.255 & 0.130 \\
\hline \multicolumn{11}{|l|}{ Gonorrhea } \\
\hline \multirow{2}{*}{ Township } & \multicolumn{10}{|l|}{ Year } \\
\hline & 2005 & 2006 & 2007 & 2008 & 2009 & 2010 & 2011 & 2012 & 2013 & 2014 \\
\hline Pike & 0.388 & 0.470 & 0.318 & 0.337 & 0.340 & 0.365 & 0.333 & 0.427 & 0.336 & 0.314 \\
\hline Washington & 0.479 & 0.542 & 0.547 & 0.571 & 0.564 & 0.573 & 0.596 & 0.595 & 0.623 & 0.554 \\
\hline Lawrence & 0.518 & 0.544 & 0.531 & 0.530 & 0.557 & 0.526 & 0.528 & 0.526 & 0.465 & 0.545 \\
\hline Wayne & 0.418 & 0.340 & 0.347 & 0.313 & 0.328 & 0.237 & 0.312 & 0.280 & 0.335 & 0.380 \\
\hline Central & 0.259 & 0.253 & 0.268 & 0.265 & 0.303 & 0.287 & 0.321 & 0.302 & 0.282 & 0.290 \\
\hline Warren & 0.376 & 0.373 & 0.331 & 0.426 & 0.360 & 0.391 & 0.326 & 0.360 & 0.385 & 0.359 \\
\hline Decatur & 0.326 & 0.490 & 0.454 & 0.325 & 0.394 & 0.265 & 0.381 & 0.360 & 0.323 & 0.341 \\
\hline Perry & 0.347 & 0.383 & 0.396 & 0.437 & 0.410 & 0.387 & 0.379 & 0.434 & 0.478 & 0.404 \\
\hline Franklin & 0.555 & 0.301 & 0.439 & 0.404 & 0.361 & 0.227 & 0.439 & 0.484 & 0.437 & 0.296 \\
\hline
\end{tabular}

${ }^{\mathrm{a}}$ Gini coefficients that are closer to equality have values closer to 0 (darker green shades) while coefficients that are closer to inequality have values closer to 1 (darker red shades). 
Table 5 Multiple comparison analysis of Gini differences for chlamydia and gonorrhea between the nine townships from 2005 to $2014^{\mathrm{a}, \mathrm{b}}$

\begin{tabular}{|c|c|c|c|c|c|c|c|c|c|}
\hline \multicolumn{10}{|l|}{ Chlamydia } \\
\hline Township & Pike & Washington & Lawrence & Wayne & Central & Warren & Decatur & Perry & Franklin \\
\hline Pike & & $<.0001 *$ & $<.0001 *$ & 0.7913 & 0.0715 & 1.0000 & 0.3129 & 1.0000 & 0.4254 \\
\hline Washington & & & 1.0000 & $<.0001 *$ & $<.0001 *$ & $<.0001 *$ & $<.0001 *$ & $<.0001^{*}$ & $<.0001^{*}$ \\
\hline Lawrence & & & & $<.0001 *$ & $<.0001 *$ & $<.0001 *$ & $<.0001 *$ & $<.0001 *$ & $<.0001 *$ \\
\hline Wayne & & & & & 0.8823 & 0.5249 & 0.9978 & 0.5504 & 0.9997 \\
\hline Central & & & & & & $0.0228^{*}$ & 0.9989 & $0.0255^{*}$ & 0.9941 \\
\hline Warren & & & & & & & 0.1353 & 1.0000 & 0.2034 \\
\hline Decatur & & & & & & & & 0.1473 & 1.0000 \\
\hline Perry & & & & & & & & & 0.2195 \\
\hline \multicolumn{10}{|l|}{ Franklin } \\
\hline \multicolumn{10}{|l|}{ Gonorrhea } \\
\hline Township & Pike & Washington & Lawrence & Wayne & Central & Warren & Decatur & Perry & Franklin \\
\hline Pike & & $<.0001^{*}$ & $<.0001 *$ & 0.8627 & $0.0194 *$ & 1.0000 & 1.0000 & 0.6657 & 0.9137 \\
\hline Washington & & & 0.8425 & $<.0001 *$ & $<.0001 *$ & $<.0001 *$ & $<.0001 *$ & $<.0001 *$ & $<.0001^{*}$ \\
\hline Lawrence & & & & $<.0001 *$ & $<.0001 *$ & $<.0001 *$ & $<.0001 *$ & $<.0001 *$ & $<.0001 *$ \\
\hline Wayne & & & & & 0.5193 & 0.7214 & 0.8209 & $0.0364 *$ & 0.1243 \\
\hline Central & & & & & & $0.0087^{*}$ & $0.0149 *$ & $<.0001 *$ & $0.0002 *$ \\
\hline Warren & & & & & & & 1.0000 & 0.8209 & 0.9747 \\
\hline Decatur & & & & & & & & 0.7214 & 0.9398 \\
\hline Perry & & & & & & & & & 0.9999 \\
\hline Franklin & & & & & & & & & \\
\hline
\end{tabular}

${ }^{\mathrm{a} A l l} \mathrm{p}$-values were tabulated using the Tukey adjustment. ${ }^{\mathrm{b}}$ Statistically significant $\mathrm{p}$-values are represented by an asterisk at $\alpha=0.05$. 


\section{DISCUSSION}

\section{Interpretation of Findings}

This study presents a method of measuring and visualizing inequality of chlamydia and gonorrhea rates longitudinally for an urbanized county using the Gini coefficient for a period of ten years. Our findings show that at the county level, the distribution of disease rates for both chlamydia and gonorrhea are not distributed uniformly. The level of inequality for the county was not extreme, yet the Gini coefficients show that disease rates are far from homogenous. This result is consistent with past literature that displayed heterogeneous patterns in disease rates for sexually transmitted diseases [37-39]. Areas of high disease rates exist, particularly in the central part of the county, while outlying areas near adjacent bordering counties have lower disease rates. The patterns of varied and uneven disease rates in the county were evident over the course of the entire ten years of study.

Additionally, our results indicate that gonorrhea has higher inequality in the distribution of disease rates than chlamydia. This was reflected in the very large percentage of the gonorrhea Gini coefficients. In some locations where disease rates were very high, the gonorrhea disease rates were more concentrated and clustered than those for chlamydia which is consistent with previous studies that indicate gonorrhea being less evenly distributed [20,38]. It may indicate that gonorrhea represented distributions containing many census tracts with low disease rates and a few census tracts with extremely high disease rates. This would portray high inequality where only an extremely small fraction of the population receives high levels of cases compared to the rest of the population.

Furthermore, our study showed that the patterns of inequality of both chlamydia and gonorrhea disease rates in the county were not drastically different from year to year. These observations were consistent as areas with concentrated high rates of disease and very low rates of disease were expected to occur in the same general location over the entire ten-year period. There was no major change in where the concentrations of high rates of disease abruptly shift or migrate towards other areas. This finding shows that high risk infection areas are endemic to specific locations within the county, and these locations that have high infection rates are ideal for localized targeted interventions.

One note of interest was that Central Township exhibited some of the lowest Gini coefficient indices despite having different magnitudes of disease rates compared to some of the southernmost townships (Decatur, Franklin) that also showed generally low Gini coefficient values. In the visualizations, Central Township was characterized as having many census tracts with moderate and high disease rates while the southernmost townships predominantly had census tracts with much lower disease rates. Both of these different locations did portray smaller inequality in disease rates than other areas of the county. From this result, it appears that using the Gini inequality measurements may not be a good indicator to differentiate or measure the magnitude of the disease rates but is sufficient to measure the dispersion of those disease rates within the townships. 
By using decile groups, our study provides a strength in understanding the characteristics of different inequality categories within our data when chlamydia and gonorrhea are compared against each other. For all ten years of study, the percent population of the top half decile groups $\left(6^{\text {th }}\right.$ to $10^{\text {th }}$ decile group) were roughly similar representing close to half of the population. However, the Gini coefficient was higher when the percentage of cases was greater within the top half of the population as exhibited for gonorrhea. Coincidently, within the $1^{\text {st }}$ decile group, the percent population were also roughly consistent across all ten years. Yet, the Gini coefficient was higher when the percentage of cases within the $1^{\text {st }}$ decile group was smaller. These results may indicate that higher percentages of cases in the highest decile groups play a role in influencing and increasing the values of Gini coefficients.

\section{Implications for Clinicians}

There is tremendous opportunity that the measurement and identification of health inequalities provide beneficial applications and advantages for clinicians. By using inequality methods such as the Gini coefficient along with spatial data visualizations, clinicians can determine and identify disparities and gaps in screening coverage in high risk locations. If a patient is asymptomatic for a sexually transmitted disease, they are more likely to not seek treatment which would propagate the disease further to other sexual partners [40]. The increasing potential for proactive screening could detect these patients ahead of time reducing the risk of complications caused by chlamydia and gonorrhea.

Another important benefit for clinicians is to generate knowledge in implementing effective targeted intervention programs. Providers may need to decide whether chlamydia or gonorrhea intervention programs over a large area would be effective or whether they may need to succinctly target localized small areas to maximize the benefit of their programs. When the inequalities were measured by geographical areas in our study, there were differences in the distribution of disease rates within townships. This could signify that specialized targeted intervention programs that are specific to the individual high-risk community or neighborhood may perhaps work more effectively than having the same program for the entire county. Knowledge of the distribution of disease rates can guide clinicians to adjust their intervention programs, create additional healthcare services, or family planning programs according to the needs of the community or whenever their current programs are not working effectively [41]. As a result, educational activities that are included in the intervention programs can be implemented to inform patients about safe sexual health practices, understand sexually transmitted disease risks, and to seek treatment [42].

\section{Implications for Public Health Organizations}

Along with clinicians, public health organizations can benefit from the knowledge gained from using the measures of inequalities for sexually transmitted diseases. First, understanding inequalities can help assess where the demand for urgent health provisions and resources should be administered. Segments of the population that are marginalized, limited to access of healthcare, or have a demand for health programs or services can be prioritized or targeted by government agencies to increase more health funding. This could greatly assist policy makers to approve targeted resources for those at-risk individuals and communities. In some instances, having localized interventions that target a small specific area could be better than comprehensive 
interventions that target a massive geographical area. Valuable health resources would be directed to focus on highly critical infectious communities and core groups to minimize further spread of sexually transmitted diseases [19]. Using inequality measures such as the Gini coefficient can determine if public health resources are properly administered. High numbers of cases that occur disproportionally in a small geographical area could suggest that existing public health services and delivery across the county are inconsistent or ineffective [43]. Knowing that public health funds are often limited, it is necessary to have appropriate allocation and proper management of services. Thus, public health organizations can use information of inequality methods to better evaluate and improve these services.

The use of inequality measures such as the Gini coefficient can assist in monitoring trends in disease patterns over time and improve longitudinal collection of data for surveillance purposes. It can also help determine where abrupt and abnormal outbreaks of infection can occur with unusual changes in disease rates over a community or geographical area. Combined with spatial data visualization techniques, this can assist in identifying how affected populations are concentrated or dispersed as well as recognizing the factors that contribute to these characteristics of inequality.

Finally, the incorporation of methods in health inequalities can enhance and improve our knowledge of data from communicable diseases. Usage of electronic health records and health information exchange systems to capture communicable disease data will continue to increase, and the ability to perform analyses on existing reported data and identifying disparities is crucial in knowing how to manage and effectively reduce the health burdens of disease. The effectiveness of using and understanding data from health information technology to develop ongoing innovative methodologies will determine how public health organizations can combat and reduce the existing gaps of health disparities that is occurring in the health of our population.

\section{Limitations}

In our study, there were some limitations that need to be considered. Our study only represented cases that were both geocoded and reported through the INPC health information exchange system. Cases that did not have an address or were not able to be geocoded were not included in this study which would limit the number of actual positive cases. There is also the issue of inaccurate reporting by providers as some providers may not send or report actual positive cases to public health. Some providers who report cases may not participate in the INPC which also reduces the positive cases recorded. Additionally, reported rates of disease are generally influenced by diagnostic, screening, and testing behaviors. When these factors are combined with the asymptomatic nature of chlamydia and gonorrhea, the true disease rates for both chlamydia and gonorrhea could be higher than what is given in our study. However, our study provides an opportunity to estimate sexually transmitted disease rates given by the information received from health information systems to record communicable disease data. As more clinicians and healthcare providers continue to use health information systems and electronic health records to capture patient data, the ability to use accurate data from these entities to produce relevant treatment interventions is vital. 
Furthermore, consideration must be made if the results can be generalized to other geographical areas that do not have the same characteristics as our urbanized county under study. These areas may include less populated rural or suburban locations with different demographics and patterns of disease. The level of chlamydia or gonorrhea inequality may not be as pronounced or similar to the findings in this study. However, the application and the concept of analyzing and using inequality measures are still important for rural areas to measure disparities in healthcare coverage and availability of health services [44].

There are potential considerations that must be addressed when performing the Gini coefficient calculations. The Gini coefficient only gives a relative measure on the magnitude of inequality in a given distribution. It does not locate or designate where inequalities occur in a given geographical area. As a result, identifying inequalities require spatial data visualization techniques to accompany the degree of disparity involved in different locations which is presented as a strength to our study. Secondly, the Gini coefficient only differentiates the characteristics of the distribution of disease rates, but it does not differentiate the magnitude of the disease rates itself. A township that has an equal distribution of high disease rates might have a similar Gini coefficient as a township that contains an equal distribution of low disease rates. In this situation, spatial data visualization again provides the benefit to visualize and compare the differences in disease rates for different geographical locations. Finally, closer inspection must be made for townships or geographical entities that contain a very small number of census tracts or aggregated features. In particular, Decatur and Franklin townships exhibited large fluctuations in Gini coefficients during the ten-year period of study which may be attributed to the instability of disease rates. Additionally, these two townships also had the fewest number of census tracts and cases compared to other neighboring townships. Caution must be given for small sample sizes as the Gini coefficient is susceptible to variation in indices when calculated over time.

\section{Future Studies}

Our work can provide opportunities for additional health disparities research. Although our study only examined the inequality of disease incidence rates for the population within the health information exchange system as a whole, new insights in information can be generated when the data can be stratified by race, gender, or age group to discover if there are differences in inequality trends over time from these groups. For example, previous studies have denoted racial segregation as a major factor in influencing inequality rates in sexually transmitted diseases [45-47]. It may be interesting to determine if the inequality in disease rates would be much higher in our townships if race was a contributor to the disease disparity.

Additionally, further investigation can be conducted to determine which factors or social determinants of health contribute to the large disparities or inequalities in disease rates. Gathering this knowledge can assist clinicians and public health organizations in understanding underlying factors such as race, economic status, unemployment, household characteristics, or the number of sexual partners that lead to increased risk of chlamydia and gonorrhea infection. More emphasis and focus can be directed on understanding how communities and neighborhood structures operate to understand disease outcomes [48]. Furthermore, investigation of the social determinants of health can identify barriers to routine screening of sexually transmitted diseases whether that be the lack of knowledge of disease symptoms or inability to pay for screening from the patient 
[49,50]. Clinicians can address these social determinants of health to determine the mechanisms and causes of why large health inequalities in sexually transmitted diseases exist in the population.

Thirdly, although we used the most basic but effective features to visualize and map inequalities using decile groups, our study can introduce other opportunities to explore additional advanced mapping techniques or even forecast where future inequalities will occur. Geovisual analytics and geostatistics, which are branches of knowledge that focuses on making inferences from visual representations and patterns from data involving geographical features, can be applied to identify interesting phenomena in the data and visualizations being presented [51]. Examples of geovisual analytical techniques include spatial autocorrelation measurements, the local indicators for spatial autocorrelation (LISA) method to detect outliers and anomalies in maps, and spatial regression methods. When used effectively, geovisual analytical tools can enhance decision support for clinicians to allow exploration, identification, and analysis of relevant information that can meet problem-solving needs [52].

Finally, the application of the Gini coefficient can be translated to determine inequality rates in other diseases that use longitudinal data. Although sufficient comprehensive data is needed especially when comparing inequality over time, the broad applicability and straightforward calculation makes the Gini coefficient an opportune choice to assess disease distributions within different population groups.

\section{CONCLUSION}

Inequality measures such as the Gini coefficient combined with spatial data visualization mapping provide a unique opportunity to visualize and measure health inequality in sexually transmitted diseases while exploring underlying patterns in longitudinal data from clinical information systems. The measurement and examination of health inequality is necessary in understanding the distributions of disease incidence to improve delivery of valuable health resources and to identify population groups who are vulnerable to increased infection. As the collection of clinical data from health information systems continue to increase, the ability to explore, investigate, and analyze trends of disease incidence will be critical in reducing health disparities, improving health quality, and creating the most effective treatment interventions to alleviate the burdens of sexually transmitted diseases.

\section{ACKNOWLEDGEMENTS}

The authors acknowledge and thank Dr. Jane Wang, from Regenstrief Institute for her support in extracting the clinical data and preparing datasets for analysis. Additionally, we would also like to thank the Regenstrief Institute for facilitating access to and use of the data from the Indiana Network for Patient Care and for providing the capital resources to pursue this research."

\section{FINANCIAL DISCLOSURE}

The research reported in this publication was supported by the Centers for Disease Control and Prevention (CDC), U.S. Department of Health and Human Services (HHS), under Contract 
Number 200-2011-42027. The content is solely the responsibility of the authors and does not necessarily represent the official views of CDC or HHS.

\section{COMPETING INTERESTS}

The authors have no competing interests to report.

\section{REFERENCES}

1. Carter-Pokras O, Baquet C. 2002. What is a" health disparity"? Public Health Rep. 117(5), 426. PubMed https://doi.org/10.1016/S0033-3549(04)50182-6

2. Braveman P. 2006. Health disparities and health equity: concepts and measurement. Annu Rev Public Health. 27, 167-94. PubMed https://doi.org/10.1146/annurev.publhealth.27.021405.102103

3. Baquet CR, Carter-Pokras O, Bengen-Seltzer B. 2004. Healthcare disparities and models for change. Am J Manag Care. 10, SP5-11. PubMed

4. Sampson UK, Kaplan RM, Cooper RS, Diez Roux AV, Marks JS, et al. 2016. Reducing health inequities in the United States: Insights and recommendations from the National Heart, Lung, and Blood Institute's Health Inequities Think Tank Meeting. J Am Coll Cardiol. 68(5), 517. PubMed https://doi.org/10.1016/j.jacc.2016.04.059

5. Truman BI, Smith KC, Roy K, Chen Z, Moonesinghe R, et al. 2011. Rationale for regular reporting on health disparities and inequalities-United States. MMWR Surveill Summ. 60(Suppl 01), 3-10. PubMed

6. Ceriani L, Verme P. 2012. The origins of the Gini index: extracts from Variabilità e Mutabilità (1912) by Corrado Gini. J Econ Inequal. 10(3), 421-43. https://doi.org/10.1007/s10888-011-9188-x

7. Althaus CL, Turner KM, Schmid BV, Heijne JC, Kretzschmar M, et al. 2011. Transmission of Chlamydia trachomatis through sexual partnerships: A comparison between three individual-based models and empirical data. $J R$ Soc Interface. 9(66), 136-46. PubMed https://doi.org/10.1098/rsif.2011.0131

8. Lee WC. 1997. Characterizing exposure-disease association in human populations using the Lorenz curve and Gini index. Stat Med. 16(7), 729-39. PubMed https://doi.org/10.1002/(SICI)1097-0258(19970415)16:7<729::AID-SIM491>3.0.CO;2-A

9. Sasagawa M, Amieux PS, Martzen MR. 2017. Health equity and the Gini index in the United States. J Clin Med Ther. 2(15), 1-4.

10. Koua EL, Kraak MJ. 2004. Geovisualization to support the exploration of large health and demographic survey data. Int J Health Geogr. 3(1), 12. PubMed https://doi.org/10.1186/1476-072X-3-12 
11. Yang TC, Shoff C, Noah AJ. 2013. Spatializing health research: What we know and where we are heading. Geospat Health. 7(2), 161. PubMed https://doi.org/10.4081/gh.2013.77

12. Lal A. 2016. Spatial modelling tools to integrate public health and environmental science, illustrated with infectious cryptosporidiosis. Int J Environ Res Public Health. 13(2), 186. PubMed https://doi.org/10.3390/ijerph13020186

13. Graham SR, Carlton C, Gaede D, Jamison B. 2011. The benefits of using geographic information systems as a community assessment tool. Public Health Rep. 126(2), 298. PubMed https://doi.org/10.1177/003335491112600224

14. Han J, Zhu L, Kulldorff M, Hostovich S, Stinchcomb DG, et al. 2016. Using Gini coefficient to determining optimal cluster reporting sizes for spatial scan statistics. Int $J$ Health Geogr. 15(1), 27. PubMed https://doi.org/10.1186/s12942-016-0056-6

15. Horev T, Pesis-Katz I, Mukamel DB. 2004. Trends in geographic disparities in allocation of health care resources in the US. Health Policy. 68(2), 223-32. PubMed https://doi.org/10.1016/j.healthpol.2003.09.011

16. Asada Y, Hurley J, Norheim OF, Johri M. 2015. Unexplained health inequality-Is it unfair? Int J Equity Health. 14(1), 11. PubMed https://doi.org/10.1186/s12939-015-0138-2

17. Tandi TE, Cho Y, Akam AJC, Afoh CO, Ryu SH, et al. 2015. Cameroon public health sector: shortage and inequalities in geographic distribution of health personnel. Int J Equity Health. 14(1), 43. PubMed https://doi.org/10.1186/s12939-015-0172-0

18. Althaus CL, Turner KM, Schmid BV, Heijne JC, Kretzschmar M, et al. 2011. Transmission of Chlamydia trachomatis through sexual partnerships: a comparison between three individual-based models and empirical data. $J R$ Soc Interface. 9(66), 136-46. PubMed https://doi.org/10.1098/rsif.2011.0131

19. Leichliter JS, Chesson HW, Sternberg M, Aral SO. 2010. The concentration of sexual behaviours in the USA: A closer examination of subpopulations. Sex Transm Infect. 86(Suppl 3), iii45-51. PubMed https://doi.org/10.1136/sti.2010.042283

20. Kerani RP, Handcock MS, Handsfield HH, Holmes KK. 2005. Comparative geographic concentrations of 4 sexually transmitted infections. Am J Public Health. 95(2), 324-30. PubMed https://doi.org/10.2105/AJPH.2003.029413

21. Moran JS, Aral SO, Jenkins WC, Peterman TA, Alexander ER. 1989. The impact of sexually transmitted diseases on minority populations. Public Health Rep. 104(6), 560. $\underline{\text { PubMed }}$

22. Mills N, Daker-White G, Graham A, Campbell R. 2006. Population screening for Chlamydia trachomatis infection in the UK: A qualitative study of the experiences of those screened. Fam Pract. 23(5), 550. PubMed https://doi.org/10.1093/fampra/cml031 
23. Mariotti SP, Pascolini D, Rose-Nussbaumer J. 2009. Trachoma: global magnitude of a preventable cause of blindness. Br J Ophthalmol. 93(5), 563-68. PubMed https://doi.org/10.1136/bjo.2008.148494

24. Mishori R, McClaskey EL, Winklerprins VJ. 2012. Chlamydia trachomatis infections: Screening, diagnosis, and management. Am Fam Physician. 86(12), 1127. PubMed

25. Mosure DJ, Berman S, Kleinbaum D, Halloran ME. 1996. Predictors of Chlamydia trachomatis infection among female adolescents: A longitudinal analysis. Am J Epidemiol. 144(10), 997. PubMed https://doi.org/10.1093/oxfordjournals.aje.a008870

26. 2014 American Community Survey 5-Year demographic and housing estimates-Marion County, Indiana. United States Census Bureau. https://factfinder.census.gov/faces/tableservices/jsf/pages/productview.xhtml?src=CF. Accessed December 19, 2018.

27. United States Census Bureau. Geographic terms and concepts - Census tract. https://www.census.gov/geo/reference/gtc/gtc_ct.html. Accessed December 21, 2018.

28. Chlamydia - Reported cases and rates of reported cases in selected metropolitan statistical areas (MSAs) in alphabetical order, United States, 2013-2017. Centers for Disease Control and Prevention. https://www.cdc.gov/std/stats17/tables/7.htm. Accessed December 3, 2018.

29. Gonorrhea - Reported cases and rates of reported cases in selected metropolitan statistical areas (MSAs) in alphabetical order, United States, 2013-2017. Centers for Disease Control and Prevention. https://www.cdc.gov/std/stats17/tables/17.htm. Accessed December 3, 2018.

30. Overhage JM, Tierney WM, McDonald CJ. 1995. Design and implementation of the Indianapolis Network for Patient Care and Research. Bull Med Libr Assoc. 83(1), 48. $\underline{\text { PubMed }}$

31. Biondich PG, Grannis SJ. 2004. The Indiana network for patient care: an integrated clinical information system informed by over thirty years of experience. J Public Health Manag Pract. 10, S81-86. PubMed https://doi.org/10.1097/00124784-200411001-00013

32. Chlamydia trachomatis infection 2010 case definition. Centers for Disease Control and Prevention. https://wwwn.cdc.gov/nndss/conditions/chlamydia-trachomatis-infection/casedefinition/2010/. Accessed November 19, 2018.

33. Law DG, Serre ML, Christakos G, et al. 2004. Spatial analysis and mapping of sexually transmitted diseases to optimise intervention and prevention strategies. Sex Transm Infect. 80(4), 294-99. PubMed https://doi.org/10.1136/sti.2003.006700

34. Indiana administrative code: Title 410 Indiana State Department of Health. Indiana General Assembly. http://www.in.gov/legislative/iac/iac_title?iact=410. Accessed December 11, 2018. 
35. Regidor E. 2004. Measures of health inequalities: Part 1. J Epidemiol Community Health. 58, 858-61. PubMed https://doi.org/10.1136/jech.2003.015347

36. Keppel K, Pamuk E, Lynch J, Carter-Pokras O, Kim I, Mays V, et al. Methodological issues in measuring health disparities. Vital Health Stat 2. Data evaluation and methods research. $2005 ;(141): 1$.

37. Patterson-Lomba O, Goldstein E, Gómez-Liévano A, Castillo-Chavez C, Towers S. 2015. Per-capita incidence of sexually transmitted infections increases systematically with urban population size: a cross-sectional study. Sex Transm Infect. 91(8), 610-14. PubMed https://doi.org/10.1136/sextrans-2014-051932

38. Chesson HW, Sternberg M, Leichliter JS, Aral SO. 2010. The distribution of chlamydia, gonorrhea and syphilis cases across states and counties in the USA, 2007. Sex Transm Infect. 86(Suppl 3), iii52-57. PubMed https://doi.org/10.1136/sti.2009.040873

39. Marotta P. 2017. Assessing spatial relationships between race, inequality, crime, and gonorrhea and chlamydia in the United States. J Urban Health. 94(5), 683-98. PubMed https://doi.org/10.1007/s11524-017-0179-5

40. Cattley C, Massari P, Genco CA. 2015. Incidence of gonorrhea and chlamydia in urban settings: The case for neighborhood level analysis in Boston. Adv Infect Dis. 5(04), 162. https://doi.org/10.4236/aid.2015.54020

41. Chen J, Roth RE, Naito AT, Lengerich EJ, MacEachren AM. 2008. Geovisual analytics to enhance spatial scan statistic interpretation: An analysis of US cervical cancer mortality. Int J Health Geogr. 7(1), 57. PubMed https://doi.org/10.1186/1476-072X-7-57

42. Rosen NO. 2005. KnÄuper B, Mozessohn L, Ho MHR. Factors affecting knowledge of sexually transmitted infection transmissibility in healthcare providers: Results from a national survey. Sex Transm Dis. 32(10), 619-24. PubMed https://doi.org/10.1097/01.olq.0000179889.61390.63

43. Hardwick D, Patychuk D. 1999. Geographic mapping demonstrates the association between social inequality, teen births and STDs among youth. Can J Hum Sex. 8(2), 77.

44. Jenkins WD, Rabins C, Bhattacharya D. 2011. Importance of physicians in Chlamydia trachomatis control. Prev Med. 53(4-5), 335-37. PubMed https://doi.org/10.1016/j.ypmed.2011.08.022

45. Thomas JC, Gaffield ME. 2003. Social structure, race, and gonorrhea rates in the southeastern United States. Ethn Dis. 13(3), 362-68. PubMed

46. Biello KB, Kershaw T, Nelson R, Hogben M, Ickovics J, et al. 2012. Racial residential segregation and rates of gonorrhea in the United States, 2003-2007. Am J Public Health. 102(7), 1370-77. PubMed https://doi.org/10.2105/AJPH.2011.300516 
47. Pugsley RA, Chapman DA, Kennedy MG, Liu H, Lapane KL. 2013. Residential segregation and gonorrhea rates in US metropolitan statistical areas, 2005-2009. Sex Transm Dis. 40(6), 439-43. PubMed https://doi.org/10.1097/OLQ.0b013e31828c6416

48. Ford JL, Browning CR. 2014. Neighborhoods and infectious disease risk: Acquisition of chlamydia during the transition to young adulthood. J Urban Health. 91(1), 136-50.

PubMed https://doi.org/10.1007/s11524-013-9792-0

49. Owusu-Edusei K, Owens CJ. 2009. Monitoring county-level chlamydia incidence in Texas, 2004-2005: Application of empirical Bayesian smoothing and exploratory spatial data analysis (ESDA) methods. Int J Health Geogr. 8(1), 12. PubMed https://doi.org/10.1186/1476-072X-8-12

50. Keegan MB, Diedrich JT, Peipert JF. 2014. Chlamydia trachomatis infection: Screening and management. J Clin Outcomes Manag. 21(1), 30. PubMed

51. Keim DA, Mansmann F, Schneidewind J, Ziegler H. Challenges in visual data analysis. In: Tenth International Conference on Information Visualisation. Zürich: IEEE; July 2006.

52. Andrienko G, Andrienko N, Jankowski P, Keim D, Kraak MJ, et al. 2007. Geovisual analytics for spatial decision support: Setting the research agenda. Int J Geogr Inf Sci. 21(8), 839-57. https://doi.org/10.1080/13658810701349011 